\title{
Fluorescence tomographic imaging of sentinel lymph node using near-infrared emitting bioreducible dextran nanogels
}

This article was published in the following Dove Press journal:

International Journal of Nanomedicine

4 December 2014

Number of times this article has been viewed

\author{
Jiejing $\mathrm{Li}^{1 *}$ \\ Beiqi Jiang ${ }^{*}$ \\ Chao Lin $^{2}$ \\ Zhigang Zhuang' \\ 'Department of Breast Surgery, \\ Shanghai First Maternity and Infant \\ Hospital, ${ }^{2}$ The Institute for Biomedical \\ Engineering and Nanoscience, Tongji \\ University School of Medicine, \\ Tongji University, Shanghai, People's \\ Republic of China \\ *These authors contributed equally \\ to this work
}

\begin{abstract}
Sentinel lymph node (SLN) mapping is a critical procedure for SLN biopsy and its diagnosis as tumor metastasis in clinical practice. However, SLN mapping agents used in the clinic frequently cause side effects and complications in the patients. Here, we report the development of a near-infrared (NIR) emitting polymeric nanogel with hydrodynamic diameter of $\sim 28 \mathrm{~nm}$ - which is the optimal size for SLN uptake - for noninvasive fluorescence mapping of SLN in a mouse. This polymeric nanogel was obtained by coupling Cy7, an NIR dye, to the self-assembled nanogel from disulfide-linked dextran-deoxycholic acid conjugate with the dextran of $10 \mathrm{kDa}$, denoted as Dex-Cy7. Fluorescence imaging analysis showed that Dex-Cy7 nanogels had an enhanced photostability when compared to $\mathrm{Cy} 7$ alone. After intradermal injection of Dex-Cy7 nanogel into the front paw of a mouse, the nanogels were able to migrate into the mouse's axillary lymph node, exhibiting longer retention time and higher fluorescence intensity in the node when compared to Cy7 alone. An immunohistofluorescence assay revealed that the nanogels were localized in the central region of lymph node and that the uptake was largely by the macrophages. In vitro and in vivo toxicity results indicated that the dextran-based nanogels were of low cytotoxicity at a polymer concentration up to $1,000 \mu \mathrm{g} / \mathrm{mL}$ and harmless to normal liver and kidney organs in mice at an intravenous dose of $1.25 \mathrm{mg} / \mathrm{kg}$. The results of this study suggest that NIR-emitting polymeric nanogels based on bioreducible dextran-deoxycholic acid conjugates show high potential as fluorescence nanoprobes for safe and noninvasive SLN mapping.
\end{abstract}

Keywords: nanogel, disulfide, dextran, lymph node, tomographic imaging

\section{Introduction}

Sentinel lymph node (SLN) biopsy has been validated as a pivotal approach for the diagnosis tumor metastasis in patients with breast cancer and melanomas. ${ }^{1,2}$ For successful SLN biopsy, an accurate mapping of SLN is required. ${ }^{3}$ Although SLN mapping tracers such as blue dyes and radioactive colloids have been used in the clinic, they have a few drawbacks. For example, radioactive agents cause radiation damage to the tissues adjacent to the SLN. ${ }^{4}$ Blue dyes display a short retention time in SLN as a result of their quick diffusion from SLN to the second-tier nodes, thereby causing false-negative diagnosis. As a result, the detection of SLN with methylene blue (MB) has to be conducted by physicians with sufficient clinical experience. Thus, there is a fundamental need to develop safe and effective tracers for noninvasive SLN mapping.

In order to achieve an ideal tracer for accurate SLN mapping, an optimal particle size is a key factor because it influences the uptake rate of the tracer by the lymph node (LN) and the subsequent retention time. Generally, the nanoparticles with
Correspondence: Chao Lin

Room 222, Shixun Building, Tongj

University, Shanghai, 200092,

People's Republic of China

Email chaolin@tongji.edu.cn

Zhigang Zhuang

Shanghai First Maternity and Infant Hospital, 536 Changle Road, Shanghai, 200040, People's Republic of China

Tel +862165988029

Fax +862165983706

Email I37lji_zzg@tongji.edu.cn 
hydrodynamic diameters of 10-50 $\mathrm{nm}$ have shown rapid uptake into the lymphatic system. ${ }^{5,6}$ Nakajima et al indicated that nanoparticles with a hydrodynamic diameter of $20 \mathrm{~nm}$ or $40 \mathrm{~nm}$ stayed in SLN for a relatively long time. ${ }^{7}$ Furthermore, for noninvasive SLN mapping, the SLN tracer must possess noninvasive imaging property and good biocompatibility. Over the past few years, near-infrared (NIR) fluorescence imaging has shown great promise for noninvasive imaging because such fluorescence has a relatively deep penetration ability in living tissue and the tissue has a weak NIR auto-fluorescence background. ${ }^{8}$ As such, a number of NIRemitting tracers were developed as fluorescence nanoprobes for noninvasive SLN mapping in animal models. ${ }^{9}$ Particularly, a few inorganic nanoprobes based on quantum dots (QDs) and silica dots for SLN mapping have received much attention due to their good photostability, easy synthesis, and versatile nanostructures. ${ }^{10,11}$ However, cytotoxicity and biocompatibility of these inorganic nanoparticles have not been thoroughly elucidated, thus hampering their further clinical practices. Alternatively, organic nanoprobes based on polymeric nanogels, nanocomplexes, and nanoliposomes have better biocompatibility because they usually consist of low-toxic and biocompatible materials such as polyethylene glycol and polysaccharides. For example, Noh et al reported on the IR dye 800 -coupled pullulan nanogels which had low cytotoxicity and were effective for SLN mapping in a mouse and a big model. ${ }^{12}$ Melancon et al showed the possibility of low-toxic NIR 813 dye-labeled poly-L-glutamic acid nanocomplexes for fluorescence mapping of SLN in a mouse model. ${ }^{13}$ However, these polymeric nanoprobes are nondegradable or biodegrade only slowly, which likely causes chronic toxicity and becomes a barrier to clinical translation.

Therefore, for safe and effective SLN mapping, it would be attractive to develop degradable polymeric nanoprobes. Recently, efforts have been made to fabricate disulfide-based (bioreducible) nanosystems for biomedical applications such as controlled drug release because of the unique feature of the disulfide bond, that is, relatively high chemical stability under an extracellular condition but biodegradability in an intracellular reducing environment. This feature thus makes the disulfide bond very valuable to generate biodegradable nanosystems that have low toxicity as compared to their nondegradable counterparts. ${ }^{14}$ Inspired by this finding, we propose in this study to design and prepare NIR-emitting biodegradable nanogels for fluorescence SLN mapping. To this end, a group of disulfide-linked dextran-deoxycholic acid conjugates (denoted as Dex-SS-DCA) were synthesized and characterized. Next, chemical coupling of $\mathrm{Cy} 7$, an NIR dye, to Dex-SS-DCA conjugate was performed to afford a bioreducible nanogel (named as Dex-Cy7) as a fluorescence nanoprobe for SLN imaging in a mouse. It is hypothesized that the Dex-Cy 7 nanogels should be biodegradable with low toxicity and good biocompatibility in vivo. Physiochemical properties of the nanogels were investigated in terms of size, surface charge, photostability, as well as in vitro and in vivo toxicity. The imaging, accumulation kinetics in the $\mathrm{LN}$, and in vivo biodistribution of Dex-Cy7 nanogels were evaluated with a fluorescence tomographic imaging system. In vitro uptake mechanism of the nanogels was also evaluated against DC 2.4 cells.

\section{Materials and methods \\ Materials}

Dextran (Dex, $M_{\mathrm{w}}=10 \mathrm{k} \mathrm{g} / \mathrm{mol}, 20 \mathrm{k} \mathrm{g} / \mathrm{mol}, 40 \mathrm{k} \mathrm{g} / \mathrm{mol}$ ), dithiothreitol (DTT), $N$-hydroxysuccinimide (NHS), 1-ethyl3-(3-dimethylaminopropyl)-carbodiimide (EDC), anhydrous dimethylformamide, deoxycholic acid (DCA), and 4',6-diamidino-2-phenylindole dihydrochloride (DAPI) were obtained from Sigma-Aldrich (Shanghai, PR China). $p$-Nitrophenyl chloroformate (PNC) was procured from ADMAS (Shanghai, PR China). Cy7-NHS was purchased from Beijing Fanbo Inc (Beijing, PR China). 2-(Pyridyldithio)-ethylamine (PDA) was synthesized according to a previous report. ${ }^{15}$

\section{Synthesis of Dex-SS-DCA linked by disulfide bond}

Dex-SS-DCA conjugates were prepared via a four-step synthesis (Figure 1). First, thiolated dextran (Dex-SH) with the substitution degree (DS, defined as the number of thiol groups per 100 anhydroglucose rings of dextran) of five was synthesized via a two-step procedure according to our previous report. ${ }^{16}$ Next, Dex-SH was reacted with PDA to give dextran-cysteamine conjugate (Dex-SS- $\mathrm{NH}_{2}$ ). Finally, Dex10k-SS- $\mathrm{NH}_{2}$ was coupled with DCA via an EDC/NHS activation reaction to yield Dex-SS-DCA. As a typical example for the synthesis of Dex10k-SS-NH ${ }_{2}$, Dex10k-SH (DS 5, $0.0125 \mathrm{mmol} \mathrm{SH}$ ) was reacted overnight with PDA $(0.25 \mathrm{mmol})$ in acidic water $(\mathrm{pH} 5,5 \mathrm{~mL})$ under nitrogen protection at room temperature. The solution was diluted with acidic water $(\mathrm{pH} \sim 5)$ and purified by ultrafiltration ( molecular weigh cut-off 1,000 ), and the dextran-cysteamine conjugate (Dex10k-SS- $\mathrm{NH}_{2}$ ) was obtained as a white powder after freeze-drying (yield: 90\%). For the synthesis of Dex10kSS-DCA, DCA $(0.25 \mathrm{mmol})$ was activated by EDC/NHS in dimethyl sulfoxide (DMSO, $1 \mathrm{~mL}$ ) for 1 hour. The DCA 


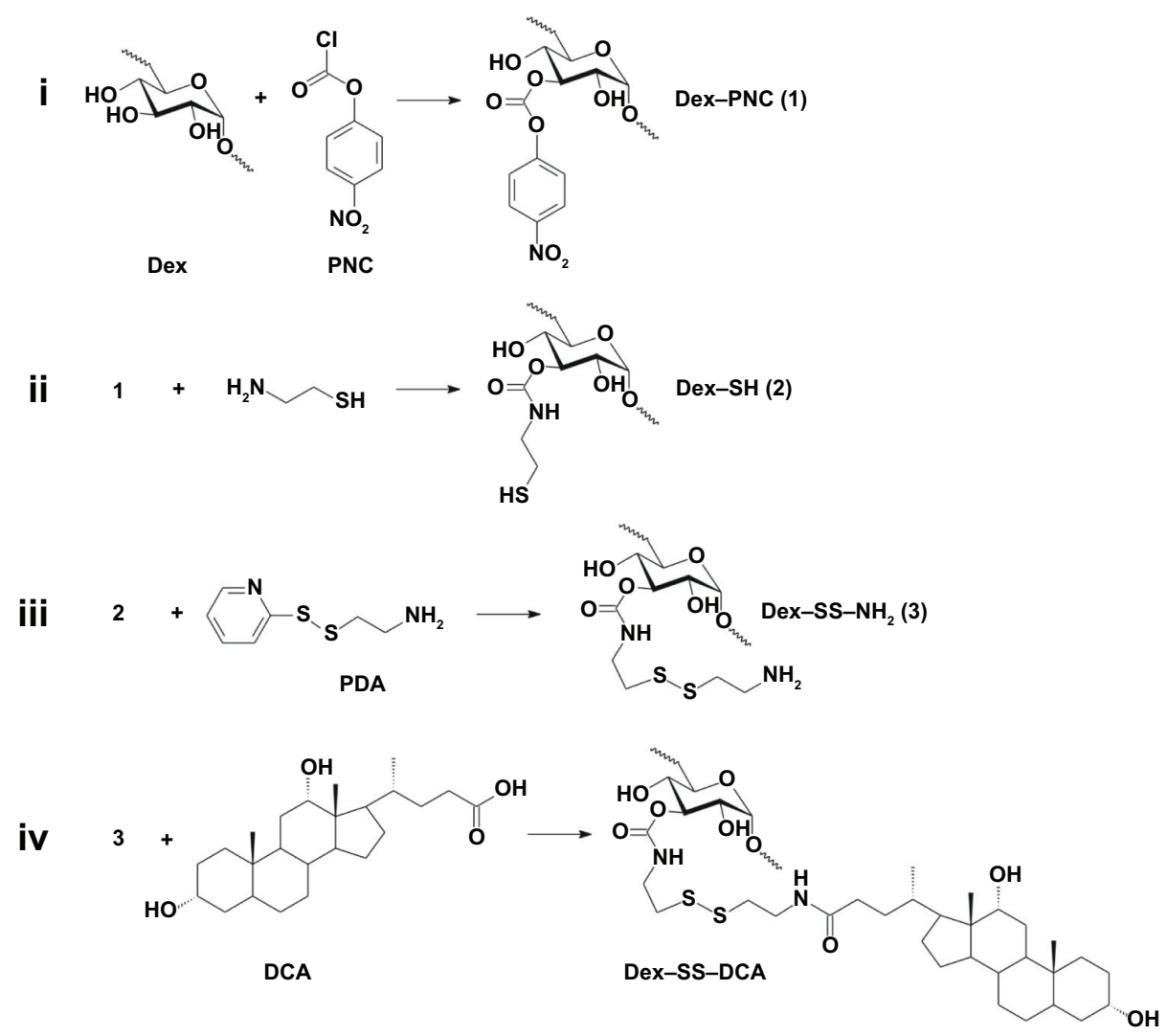

Figure I Synthesis of dextran-deoxycholic acid conjugates linked by disulfide bond (Dex-SS-DCA).

Note: Reaction steps are denoted by i-iv.

Abbreviations: PDA, 2-(pyridyldithio)-ethylamine; Dex-PNC, p-nitrophenyl chloroformate-coupled dextran; Dex-SH, thiolated dextran; Dex-SS-NH2, dextran-cysteamine conjugate; Dex-SS-DCA, disulfide-linked dextran-deoxycholic acid conjugate.

solution was reacted with Dex10k-SS- $\mathrm{NH}_{2}(0.125 \mathrm{mmol}$ $\left.\mathrm{NH}_{2}\right)$ and pyridine $(0.25 \mathrm{mmol})$ in deionized water $(0.2 \mathrm{~mL})$ for 2 days under nitrogen protection at room temperature. The residue mixture was purified by dialysis in ethanol $(3 \times 500 \mathrm{~mL})$, and the precipitate was dissolved in DMSO $(2 \mathrm{~mL})$ for exhaustive dialysis in deionized water $(3 \times 5 \mathrm{~L})$. Finally, Dex10k-SS-DCA was obtained as a white solid powder after freeze-drying. ${ }^{1} \mathrm{H}$ NMR of Dex10k-SS-NH $\left(\mathrm{D}_{2} \mathrm{O}, \delta\right): 2.8-3.0\left(4 \mathrm{H}, \mathrm{C}_{2} \mathrm{SSC}_{2}\right), 3.37\left(4 \mathrm{H}, \mathrm{C}_{2} \mathrm{CH}_{2} \mathrm{SS}-\right.$ $\mathrm{CH}_{2} \underline{\mathrm{C}}_{2} \mathrm{NH}_{2}$ ), 5.0 (s, $1 \mathrm{H}$, dextran anomeric proton). ${ }^{1} \mathrm{H} \mathrm{NMR}$ of Dex10k-SS-DCA $\left(\mathrm{D}_{2} \mathrm{O}, \delta\right): 0.58\left(3 \mathrm{H}, 8-\mathrm{CH}_{3}\right), 0.84(1 \mathrm{H}$, 7- $\left.\mathrm{CH}_{3}\right), 0.90\left(1 \mathrm{H}, 6-\mathrm{CH}_{3}\right), 5.0(\mathrm{~s}, 1 \mathrm{H}$, dextran anomeric proton), as shown in Figure 2.

\section{Synthesis of Cy7-labeled Dex-SS-DCA (Dex-Cy7) nanoprobe}

For the preparation of Dex-Cy7 nanoprobe, Dex-SS-DCA (15 mg) was reacted with Cy7-NHS (45 $\mu \mathrm{L}$ in DMSO, $10 \mathrm{nmol} / \mu \mathrm{L}$,$) in anhydrous DMSO (200 \mu \mathrm{L})$ as a solvent. The coupling reaction proceeded for 1 day at room temperature. The resulting solution was then purified by ultrafiltration in deionized water. Rhodamine B-labeled Dex-SS-DCA nanogel (Dex-RB) was also prepared.

\section{Physiochemical characterization}

${ }^{1} \mathrm{H}$ NMR spectra were recorded on a BRUKER AV-500 (Bruker, MA, USA) spectrometer equipped with a 5-mm TXO probe head and nominal frequency of $500 \mathrm{MHz}$ (number of scans: 64$)$. The sample ( $10 \mathrm{mg}$ ) was prepared and dissolved in $0.8 \mathrm{~mL}$ of $\mathrm{D}_{2} \mathrm{O}$ or DMSO. The signal of the solvent residue was used as a reference peak for the ${ }^{1} \mathrm{H}$ NMR chemical shift and was set at $\delta 4.79$ for $\mathrm{D}_{2} \mathrm{O}$ and $\delta 2.5$ for DMSO. The particle size and zeta potential of DexSS-DCA nanogels were measured at $25^{\circ} \mathrm{C}$ with Nanosizer NS90 (Malvern Instrument, Malvern, UK). Thermogravimetric analysis of Dex-SS-DCA nanogels was conducted by the TGA/DSC Simultaneous Thermal Analyzer (TGA/ DSC 1/1600HT; Mettler-Toledo Co, Zurich, Switzerland). Morphology of the nanogels was observed by transmission electron microscopy (TEM) (JEM-1230; JEOL, Tokyo, Japan). The amount (in picomole) of $\mathrm{Cy} 7$ was determined by a UV-vis spectrophotometer (Bruker) at $750 \mathrm{~nm}$ wavelength 

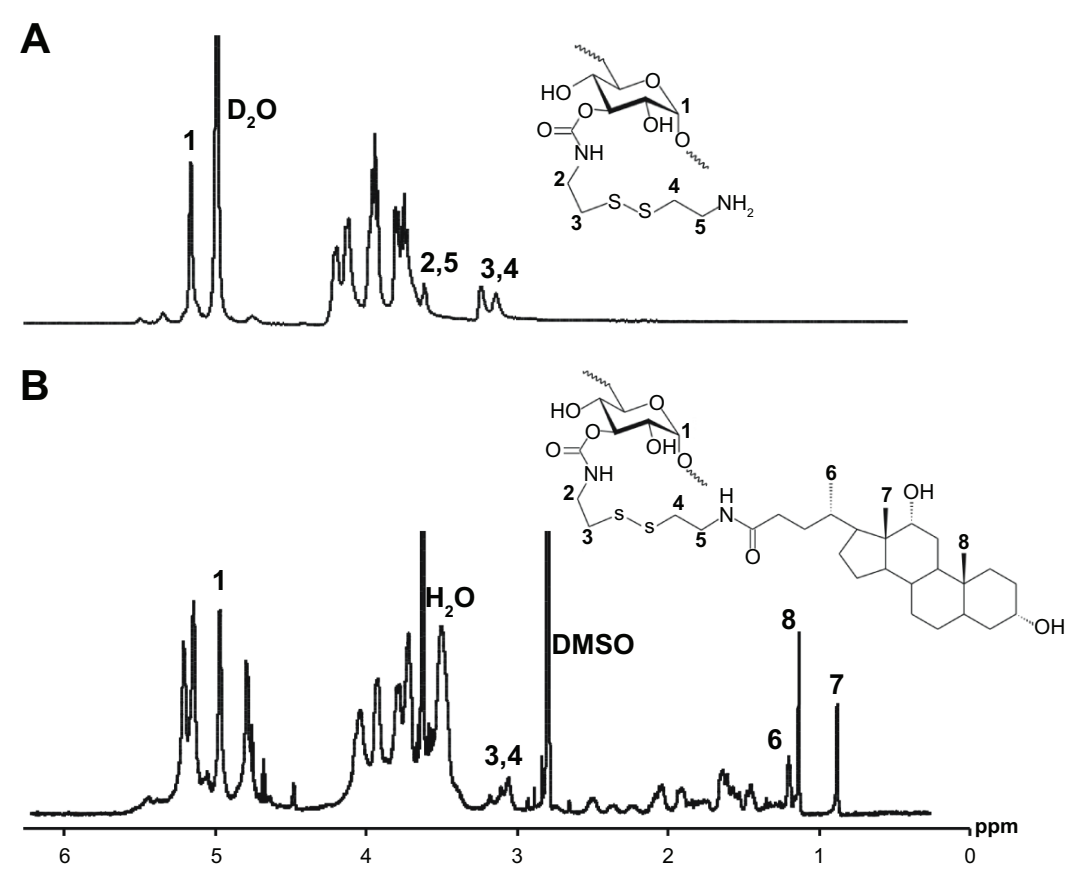

Figure 2 ' $\mathrm{H}$ NMR spectrum of (A) Dex-SS- $\mathrm{NH}_{2}$ and (B) Dexl0k-SS-DCA.

Abbreviations: Dex-SS-NH , dextran-cysteamine conjugate; Dex-SS-DCA, disulfide-linked dextran-deoxycholic acid conjugate; DMSO, dimethyl sulfoxide; ppm, parts per million; $\mathrm{D}_{2} \mathrm{O}$, deuterium oxide.

using a calibration curve based on a series of Cy7 solutions at varying concentrations.

\section{In vitro imaging and photostability of Dex-Cy7 nanoprobe}

Fluorescence imaging with Dex-Cy7 nanoprobe was performed by an $\mathrm{FMT}^{\circledR} 2000$ tomographic imaging system (PerkinElmer Inc, Waltham, MA, USA) equipped with a laser source $\left(35 \mathrm{~mW} / \mathrm{cm}^{2}\right)$ at an excitation wavelength of $745 \mathrm{~nm}$ and a charge-coupled device detector at an emission wavelength of 770-800 nm. To prove that Dex-Cy7 served as a fluorescence nanoprobe, Dex-Cy7 solutions in phosphate buffered saline (PBS) buffer (20 mM, pH 7.4) with different amounts of $\mathrm{Cy} 7$ from 5 pmol to 100 pmol were placed in a cuvette or tube and imaging was then performed. As a control, Cy7 solution with the same amount of Cy7 was also applied for imaging. To quantify fluorescence intensity (in picomole), the fluorescence-emission region was collected and calculated by a reconstruction software (True QuantTM 3D, version 2.0.0.19) provided by the manufacturer (PerkinElmer). The fluorescence photostability of Dex-Cy7 and $\mathrm{Cy} 7$ were compared at the same concentration $(100 \mathrm{pmol} / \mathrm{mL})$ and the same time intervals in the range 0.5-24 hours using the FMT 2000 system. The fluorescence intensity detected at 0 hour (as control) was set as 1.0 , and the fluorescence intensities at different time intervals were normalized to the control and plotted as a function of time.

\section{In vitro cytotoxicity assay}

MCF-7 and DC 2.4 cells (American Type Culture Collection [ATCC], Manassas, VA, USA) were incubated in full cell culture medium (ie, DMEM supplemented with 10\% FBS, $100 \mathrm{U} / \mathrm{mL}$ penicillin and streptomycin [Thermo Fisher Scientific, Waltham, MA, USA]) at $37^{\circ} \mathrm{C}$ and in $5 \% \mathrm{CO}_{2}$. In vitro cytotoxicity of the Dex-SS-DCA nanoprobe was evaluated by assay of the cell viability. In brief, MCF-7 or DC 2.4 cells $\left(1 \times 10^{4}\right.$ cells/well $)$ were seeded in a 96 -well plate and cocultured with the nanoprobe at polymer concentrations from $50 \mu \mathrm{g} / \mathrm{mL}$ to $1,000 \mu \mathrm{g} / \mathrm{mL}$ in full DMEM culture medium containing $10 \%$ FBS for 24 hours or 48 hours. Afterward, the cells were washed twice with fresh $1 \times$ PBS and cell viability was detected by AlamarBlue assay (Thermo Fisher Scientific, Waltham, MA, USA) according to the manufacturer's protocol. The value for untreated cells (as blank) was taken as $100 \%$ cell viability.

\section{In vivo biocompatibility of Dex-SS-DCA by liver and kidney function test and tissue H\&E staining}

The animal experiments were officially approved by the IACUC of Tongji University. Six-week-old male BALB/c nude mice were ordered from Shanghai Laboratory Animal Center(SLAC, Shanghai, PR China). Four-week-old Kunming mice were ordered from SLAC and divided into two groups with six mice in each group. The mice were administered 
(intravenously) with a dose of $1.25 \mathrm{mg}$ of Dex $10 \mathrm{k}-\mathrm{SS}-\mathrm{DCA}$ per $\mathrm{kg}(10 \mathrm{kDa}$ of dextran at the DS of 5). After 3 days or 14 days after injection, the mice were sacrificed. PBS injection was also used for the control group. The blood of mice was collected and the biomarkers in plasma related to liver and kidney functions, ie, aspartate transaminase, alanine transaminase, alkaline phosphatase, blood urea nitrogen, uric acid, and creatinine clearance rate, were detected by routine blood analysis. The harvested organs were fixed in 10\% formalin and embedded in paraffin wax, and the sections $(10 \mu \mathrm{m}$ depth) were stained by hematoxylin and eosin (H\&E).

\section{In vivo 3D tomographic fluorescence lymph node mapping}

Kunming mice were anesthetized with $300 \mu \mathrm{L}$ of $4 \%$ chloral hydrate (Sigma-Aldrich) in PBS solution. Hair was removed from the axillary region with a depilatory cream. Then, $30 \mu \mathrm{L}$ of PBS solution containing Dex-SS-DCA nanoprobe (1,500 pmol of Cy7) or Cy7 alone (1,500 pmol) was intradermally injected into the left forepaw pad of the mice. Fluorescence images of the LN of mice was acquired by the FMT 2000 system at an excitation and emission wavelength of $745 \mathrm{~nm}$ and $770-800 \mathrm{~nm}$, respectively. To confirm that the fluorescence image was the LN contour, Dex-Cy7 nanoprobe solution ( $30 \mu \mathrm{L}$ of PBS) was in situ injected into the left LN of the mouse after removal of the skin. The same solution was also intradermally injected into the right forepaw pad of the mouse. After 1 hour of injection, three-dimensional (3D) tomographic imaging of the left and right LNs were acquired by the FMT 2000 system. A tomographic image of LN was also analyzed in the transverse $(z)$ and axial $(x, y)$ cross sections. Also, ex vivo imaging of the left and right LNs as well as fat (as a control) was acquired for double-checking.

\section{Accumulation kinetics in lymph node and biodistribution of Dex-Cy7 nanoprobe}

Accumulation kinetics of Dex-SS-DCA nanoprobe in the LN was examined by quantification of $\mathrm{Cy} 7$ fluorescence intensity in the LN after intradermal injection of the nanoprobe $(1,500$ pmol Cy7) in PBS $(30 \mu \mathrm{L})$ into the left forepaw pad of the mice. Accumulation kinetics of $\mathrm{Cy} 7$ (1,500 pmol), as a control, was also examined with the same protocol. The amount (pmol) of Cy7 in LN were shown as a function of time ranging from 30 minutes to 3 days ( $n=3$ per group). The organs of mice were harvested 3 days after injection of the nanoprobe and its biodistribution was imaged by ex vivo imaging of the organs using the FMT 2000 system.

\section{In situ histofluorescence assay}

Biodistribution of Dex-RB in LN was analyzed by dissecting the axillary LN 2 hours after injection of the nanoprobe (1,500 pmol) and embedding in Tissue-Tek OCT (Sakura, Tokyo, Japan), followed by freezing at $-80^{\circ} \mathrm{C}$. Cryosections $(5 \mu \mathrm{m})$ were prepared with a freezing microtome (Leica Microsystems Inc, Buffalo Grove, IL, USA) and transferred to glass slides. The sections were fixed with $4^{\circ} \mathrm{C}$ acetone for 10 minutes, washed twice with $1 \times$ PBS, and blocked with $1 \times$ PBS having $5 \%$ goat serum for 15 minutes at $37^{\circ} \mathrm{C}$. The sections were stained with rat anti-mouse CD68 (1:25; Bio-Rad Laboratories Inc, Hercules, CA, USA) and rat anti-mouse CD205 (1:25, Bio-Rad Laboratories Inc) overnight at $4^{\circ} \mathrm{C}$ to label the macrophages and dendritic cells, respectively. Next, the sections were washed twice with $1 \times$ PBS and stained with FITC-labeled goat anti-rat IgG secondary antibody (1:64, Bio-Rad Laboratories Inc) for 40 minutes at $37^{\circ} \mathrm{C}$. Finally, the sections were washed twice with fresh $1 \times$ PBS and stained with DAPI (1\% in PBS) for 10 minutes. After the final washing, the sections were observed by a confocal laser scanning microscope (CLSM, Nikon A1R; Nikon Corporation, Tokyo, Japan).

\section{Intracellular trafficking of Dex-Cy7 nanoprobe}

DC 2.4 cells $\left(5 \times 10^{4}\right)$ were seeded in a 24 -well microscopy cover glass (VWR International, Radnor, PA, USA) for 24 hours at $37^{\circ} \mathrm{C}$ and in $5 \% \mathrm{CO}_{2}$. Rhodamine-B-labeled DexSS-DCA conjugate (Dex-RB) at a polymer concentration of $0.1 \mathrm{mg} / \mathrm{mL}$ was cocultured with the cells at different time intervals. After washing twice with $1 \times$ PBS, the cells were fixed with cold acetone for 20 minutes at room temperature and stained with DAPI (1\%) in PBS for 10 minutes. After washing again with $1 \times$ PBS, DC 2.4 cells were stained with $50 \mathrm{nM}$ of LysoTracker Green DND-26 (Thermo Fisher Scientific, Waltham, MA, USA) for 45 minutes. The nanoprobe in the cells was observed by CLSM.

MCF-7 and DC 2.4 cells (ATCC) were incubated in full cell culture medium (ie, DMEM supplemented with 10\% FBS, $100 \mathrm{U} / \mathrm{mL}$ penicillin and streptomycin) at $37^{\circ} \mathrm{C}$ and in $5 \% \mathrm{CO}_{2}$. For endocytosis study of Dex-RB nanogel, DC 2.4 cells $(5 \times 104)$ were seeded in a 24 -well plate for 24 hours at $37^{\circ} \mathrm{C}$ and in $5 \% \mathrm{CO}_{2}$. After the cells were treated by the inhibitors (chlorpromazine, wortamannin, and $\beta$-cyclodextrin) at different concentrations for 1 hour, Dex-RB nanogels at a concentration of $0.1 \mathrm{mg} / \mathrm{mL}$ were cocultured with the cells for another hour. Afterward, the cell lysates were collected and their fluorescence intensity was recorded at the excitation 
and emission wavelengths of $488 \mathrm{~nm}$ and $530 \mathrm{~nm}$, respectively. The intensity was normalized to the cells without inhibitor treatment as control (which is set as 100\%).

\section{Statistical analysis}

Analysis was performed using the Statistical Program for Social Science (SPSS version 9.0) for Windows XP. Differences were considered to be statistically significant at $P<0.05$ (Student's $t$-test).

\section{Results}

\section{Synthesis and characterization of} Dex-SS-DCA linked by disulfide bond

Dex-SS-DCA were synthesized via a four-step protocol (Figure 1). First, according to the method reported previously, ${ }^{16} p$-nitrophenyl chloroformate-coupled dextran (Dex-PNC) with the DS of 5 was prepared by activating hydroxyl groups of dextran with PNC in anhydrous dimethylformamide. Three Dex-PNC conjugates were produced with different molecular weights of dextran $\left(M_{\mathrm{w}}=10 \mathrm{k} \mathrm{kg} / \mathrm{mol}, 20 \mathrm{k} \mathrm{kg} / \mathrm{mol}\right.$, and $40 \mathrm{k} \mathrm{kg} / \mathrm{mol}$, respectively). Subsequently, Dex-SH was obtained by reacting an excess amount of cysteamine with these Dex-PNC conjugates. In the third step, an exchange reaction between the thiol groups of Dex-SH and PDA was conducted to afford cysteamine-dextran (Dex-SS- $\mathrm{NH}_{2}$ ) conjugates. ${ }^{1} \mathrm{H}$ NMR analysis of Dex-SS-NH indicated that their chemical shift was in good accordance with expected chemical structures. Figure 2A shows the ${ }^{1} \mathrm{H}$ NMR spectra of Dex $10 \mathrm{k}-\mathrm{SS}-\mathrm{NH}_{2}$. The DS of cysteamine residues could be calculated as $\sim 5$ by comparing the integrals of the peaks at $\delta 2.8-3.0$ $\left(\mathrm{CH}_{2} \mathrm{SSCH}_{2}\right.$ in cysteamine) and at 5.0 (dextran anomeric proton). This result implies that the thiol groups are consumed totally in the reaction. Finally, the Dex-SS-DCA was obtained via an EDC/NHS coupling reaction between DCA and Dex-SS- $\mathrm{NH}_{2}$ in $\mathrm{H}_{2} \mathrm{O} / \mathrm{DMSO}$ solvent. This solvent mixture was applied to dissolve both DCA and Dex$\mathrm{SS}-\mathrm{NH}_{2}$ and minimize the coupling of DCA to the $\mathrm{OH}$ groups of dextran. Dex-SS-DCA was obtained as a solid powder after dialysis and freeze-drying. ${ }^{1} \mathrm{H}$ NMR spectra of the Dex10k-SS-DCA conjugate showed new signals at $\delta 0.58,0.84$, and 0.90 , attributable to methyl protons of the DCA residue (Figure 2B). Moreover, from this spectra, the DS of the DCA residue in the conjugate was calculated to be $\sim 4.5$ by comparing the integrals of the peaks at $\delta 0.58$ (methyl protons of DCA) and 5.0 (anomeric proton of dextran). Table 1 gives DS values of the DCA residue of three Dex-SS-DCA conjugates, and all their DS values
Table I Characterization of Dex-SS-DCA conjugates and their nanogels

\begin{tabular}{llllll}
\hline No & Code $^{\mathrm{a}}$ & DS & $\begin{array}{l}\mathbf{C M C}^{\mathrm{b}} \\
(\mathbf{m g} / \mathbf{L})\end{array}$ & $\begin{array}{l}\text { Size }^{\mathrm{c}} \\
(\mathbf{n m})\end{array}$ & $\begin{array}{l}\zeta^{\mathrm{c}} \\
(\mathbf{m V})\end{array}$ \\
\hline I & DexI0k-SS-DCA & 4.5 & 17 & $26.3 \pm 0.5$ & $4.2 \pm 0.2$ \\
2 & Dex20k-SS-DCA & 4.8 & 32 & $35.9 \pm 0.2$ & $3.8 \pm 0.3$ \\
3 & Dex40k-SS-DCA & 4.6 & 45 & $190.7 \pm 4.5$ & $3.8 \pm 0.3$ \\
\hline
\end{tabular}

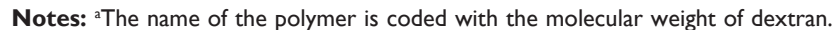
${ }^{b}$ Critical micelle concentrations were measured with the pyrene as a fluorescence probe. ${ }^{c} \zeta$ means zeta-potential. The size and $\zeta$ were measured by dynamic light scattering at a polymer concentration of $500 \mathrm{mg} / \mathrm{L}$.

Abbreviations: CMC, critical micelle concentration; DS, substitution degree; Dex-SS-DCA, dextran-deoxycholic acid conjugate.

are close to 5. Overall, these results indicate the successful preparation of Dex-SS-DCA conjugates.

\section{Characterization of Dex-SS-DCA nanogels and NIR-emitting nanogels}

The nanogels of the Dex-SS-DCA conjugates were prepared by a dialysis method. To confirm the formation of the nanogels, their particle sizes were measured by the dynamic light scattering (DLS) technique. As shown in Figure 3A, the nanogels normally had a single model size distribution with a narrow polydispersity index $(\sim 0.25)$. The surface charges of the nanogels were close to neutral (Table 1). Further, the $M_{\mathrm{w}}$ of dextran had an effect on the particle size of the Dex-SS-DCA-based nanogels. When the $M_{\mathrm{w}}$ was increased from $10 \mathrm{k}$ to $40 \mathrm{k}$, the average particle size of the nanogels increased from $26.3 \mathrm{~nm}$ to $190.7 \mathrm{~nm}$ at the same polymer concentration of $500 \mathrm{mg} / \mathrm{L}$. A rational explanation on this size increment tendency is that Dex $40 \mathrm{k}-\mathrm{SS}-\mathrm{DCA}$ is more hydrophilic than the others, thus displaying a larger particle size. Besides, using pyrene as a fluorescence probe, Dex-SSDCA conjugates had critical micelle concentrations (CMCs) in the range $17-45 \mathrm{mg} / \mathrm{L}$ (Table 1), indicating the presence of a hydrophobic area in the nanogels. TEM characterization also confirmed the formation of the nanogels with spherical morphology (see the insert in Figure 3B). Thermogravimetric analysis of Dex 10k-SS-DCA nanogels showed that they had $<5 \%$ water content and good thermostability below $100^{\circ} \mathrm{C}$ (Figure S2). These results indicate the successful formation of Dex-SS-DCA-based nanogels. Because the nanogel of Dex10k-SS-DCA has the particle size of $26.3 \pm 0.5 \mathrm{~nm}$, which is the optimal size for LN uptake, it was used in further study.

NIR-emitting nanogels were prepared by chemical conjugation of Cy7-NHS to unreacted primary amino groups of Dex10k-SS-DCA (Figure S1), being denoted as Dex-Cy7. DLS analysis revealed that Dex-Cy7 nanogels 
A

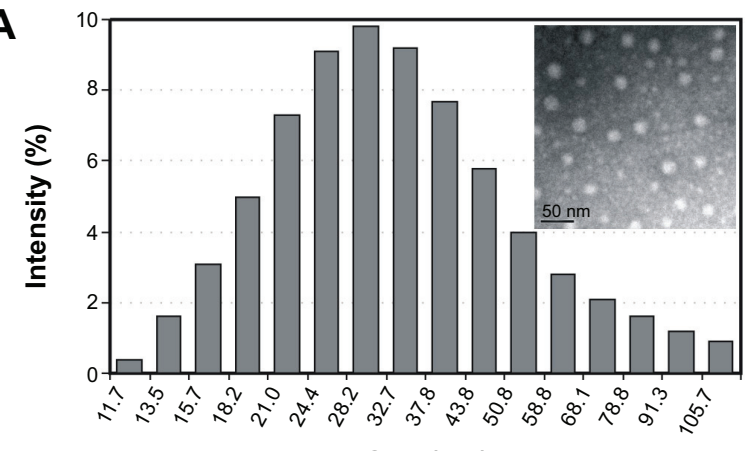

Size $(n m)$

C

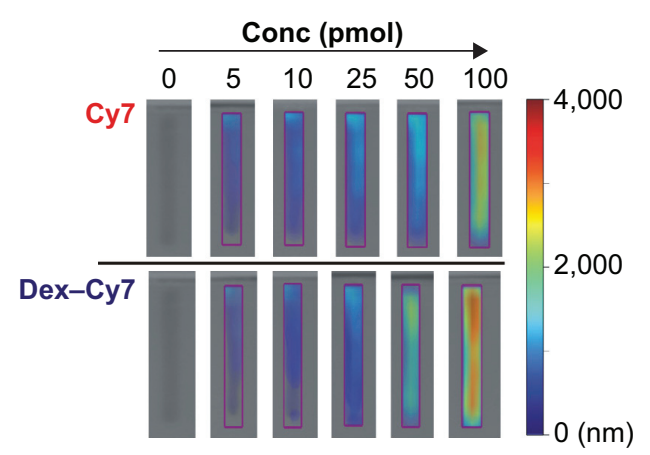

E

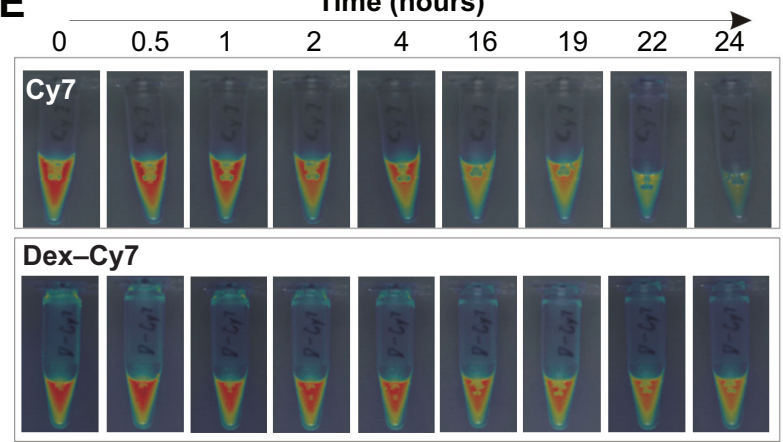

B

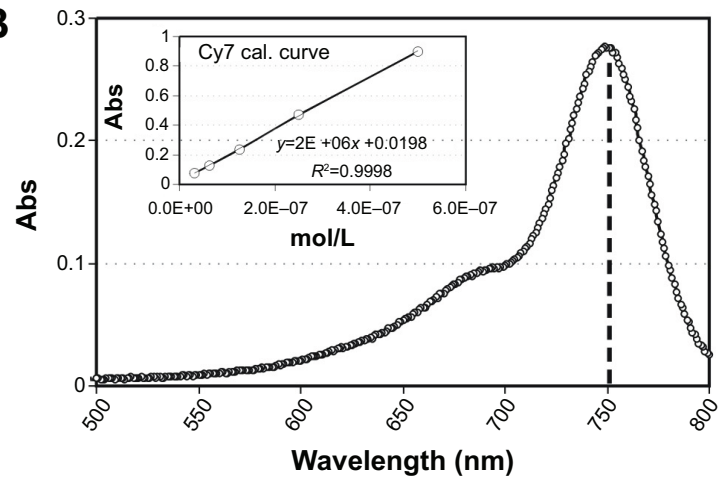

D

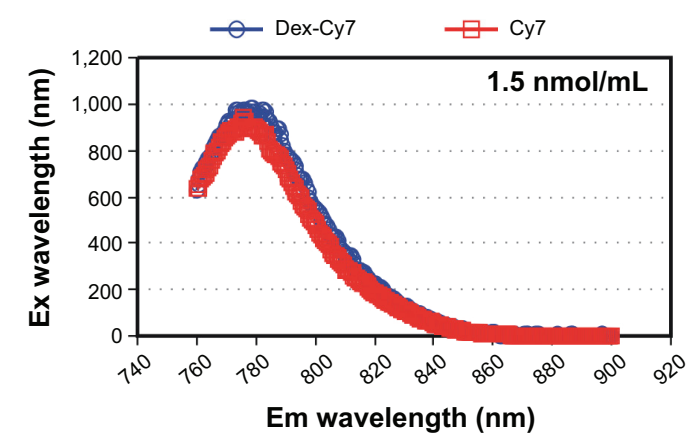

F

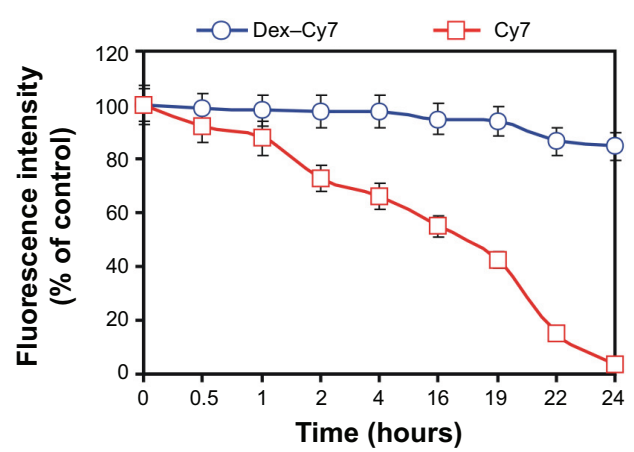

Figure 3. Characterization of bioreducible dextran-based nanogels.

Notes: (A) Size distribution of Dexl0k-SS-DCA nanogels (the insert is the TEM image of nanogel, scale bar: $50 \mathrm{~nm}$ ). (B) UV-vis spectrum of Dex-Cy7. (C) Imaging of Cy7 alone or Dex-Cy7 nanogel solution placed in a cuvette at the same amount of Cy7. (D) Fluorescence spectrum of Dex-Cy7 and Cy7 at the same Cy7 concentration of $1.5 \mathrm{nmol} / \mathrm{mL}$ at an excitation wavelength of $550 \mathrm{~nm}$. (E) Imaging of Cy7 or Dex-Cy7 nanogel solution in a tube at different time intervals in the range from 0 (as a control) to 24 hours. (F) Photostability analysis of Cy7 and Dex-Cy7 solutions by plotting fluorescence intensity as a function of time.

Abbreviations: Abs, absorbance; Conc, concentration; Ex, excitation; Em, emission; Dex-SS-DCA, dextran-deoxycholic acid conjugate; TEM, transmission electron microscopy.

had hydrodynamic diameter of $28.5 \pm 0.5$ and a neutral surface charge $(+3.6 \pm 0.2 \mathrm{mV})$. Besides, the nanogels displayed good physical stability with stable particle size within 48 hours at room temperature (Figure S3A). However, after the nanogels were treated by DTT $(2.5 \mathrm{mM})$, a thiol-containing reagent, their size rapidly enlarged to $\sim 250 \mathrm{~nm}$ with a polydispersity index of $\sim 0.5$ (Figure S3B), implying that the nanogels can be biodegradable by the disulfide cleavage in the intracellular reducing environment. In previous reports, ${ }^{17,18}$ disulfidebased nanomicelles were also found to be degradable in the presence of DTT. It is expected that this biodegradation property will contribute to the low toxicity of the nanogels (Figure 4).

UV-vis spectrum analysis showed that Dex-Cy7 nanogels had an absorbance peak at the wavelength of $750 \mathrm{~nm}$ and conjugated $\sim 43$ pmol of $\mathrm{Cy} 7$ per $1 \mathrm{mg}$ of nanogel (Figure 3B). Fluorescence imaging of the nanogel solutions showed that, at the same Cy 7 concentrations of 5-100 pmol, Dex-Cy7 nanogels afforded almost the same fluorescence images as with $\mathrm{Cy} 7$ alone (Figure 3C). This is due to the fact that the nanogel and $\mathrm{Cy} 7$ itself have the same fluorescence emission spectrum, as measured by fluorescence spectrophotometer 
(Figure 3D). These results imply that chemical coupling of $\mathrm{Cy} 7$ to dextran-based nanogel does not affect the emission spectrum of Cy7.

Figure $3 \mathrm{E}$ shows the fluorescence intensity of Dex-Cy 7 and $\mathrm{Cy} 7$ as a function of time. It was found that, with increasing time from 0.5 hour to 24 hours, the Cy 7 solution displayed weaker and weaker fluorescence intensities. For example, at 24 hours, the fluorescence intensity of the solution markedly decreased by $95 \%$ (Figure 3F). By contrast, the Dex-Cy7 nanogel solution exhibited almost the same fluorescence image within 24 hours and the fluorescence intensity only slightly reduced by $\sim 5 \%$ after 24 -hour exposure. Thus, the photostability of $\mathrm{Cy} 7$ can be improved by coupling of $\mathrm{Cy} 7$ to dextran-based nanogels.

\section{Cytotoxicity evaluation of Dex-SS-DCA nanogels}

Before imaging agents are applied for the in vivo imaging application, their toxicity properties must be elucidated. In vitro cytotoxicity of Dex-SS-DCA nanogels was tested against DC 2.4 or MCF-7 cells after they were incubated with the nanogels at concentrations from $50 \mu \mathrm{g} / \mathrm{mL}$ to $1,000 \mu \mathrm{g} / \mathrm{mL}$ within 24 hours or 48 hours. As shown in Figure 4A and B, the nanogels had low cytotoxicity with almost $100 \%$ cells maintaining survival. This low cytotoxicity may be ascribed to the good biocompatibility of dextran as well as biodegradability of the nanogels. In vivo cytotoxicity of the nanogels was also evaluated by detecting the liver and kidney function of mice 3 days or 14 days after intravenous injection of the nanogels at the dose of $1.25 \mathrm{mg} / \mathrm{kg}$. Compared to the untreated group (control), the treated mice displayed normal liver and kidney functions (Figure S4), indicating good biocompatibility of the nanogels. H\&E staining results further confirmed that the nanogels were essentially harmless to liver and kidney (Figure 4C and D). Taken together, these data show that Dex-Cy7 nanogels have low toxicity and are applicable for in vivo test.

\section{In vivo SLN mapping in mice using Dex-Cy7 nanogels}

We evaluated the Dex-Cy7 nanogel as a nanoprobe for SLN mapping by the fluorescence imaging system after intradermal injection of the nanogel solution (1,500 pmol Cy7, $30 \mu \mathrm{L}$ in PBS at $\mathrm{pH}$ 7.4) into the left paw of a mouse. Figure 5A shows in vivo fluorescence image detected in the axillary LN site of the mouse 2 hours after the injection. In order to confirm that the fluorescence signal was coming from LN, ex vivo imaging of the left LN was carried out and the fluorescence signal was observed again in the LN (Figure 5B). In order to further confirm the effectiveness of Dex-Cy7 nanogels for LN mapping, we in situ injected the nanogel solution into the left LN of a mouse after removal of the skin (Figure 5C) and, subsequently, injected the same solution into the right paw of the mouse. In this case, we observed similar fluorescence images in the left and right axillary LN sites. Also, ex vivo imaging of these two LNs confirmed that the fluorescence signals indeed were emitted from the LNs (Figure 5D). We also checked MB as a safe
A

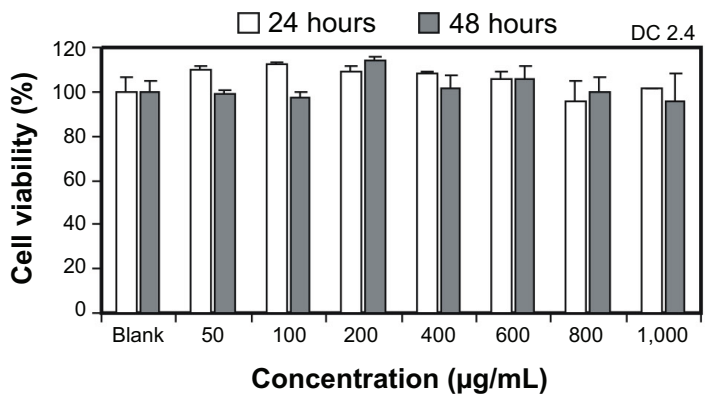

C
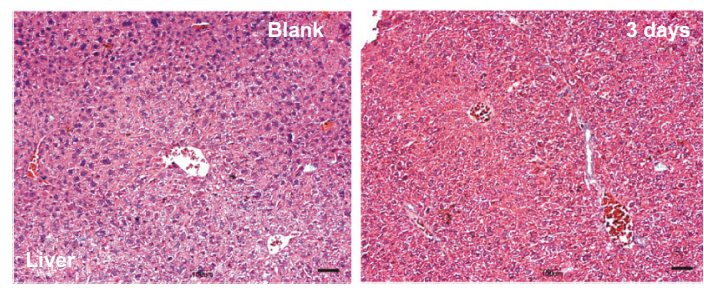

B

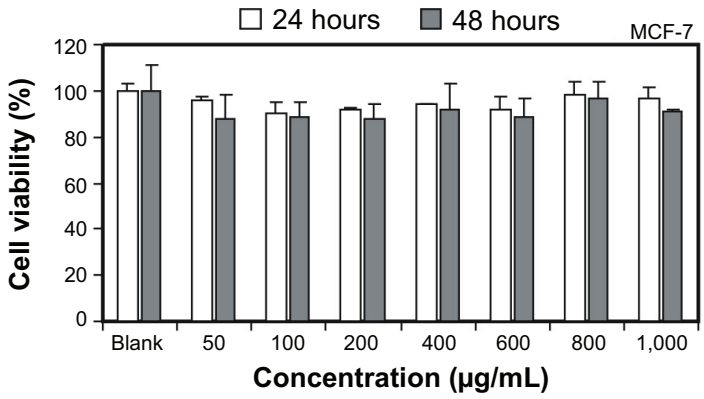

D

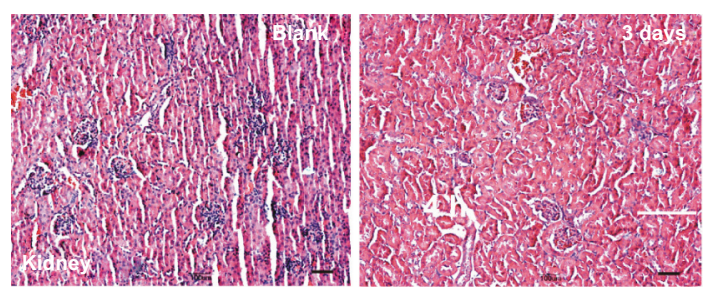

Figure $4 \mathrm{In}$ vitro and in vivo toxicity test of Dex-Cy7 nanogels.

Notes: Cytotoxicity of Dex-Cy7 nanogels by testing cell viability of (A) DC 2.4 and (B) MCF-7 cells after they are exposed in different polymer concentrations. H\&E staining of (C) liver or (D) kidney tissue 3 days after intravenous injection of Dex-Cy7 nanogel solution into a mouse at a dose of $1.25 \mathrm{mg} / \mathrm{kg}$.

Abbreviation: H\&E, hematoxylin and eosin. 

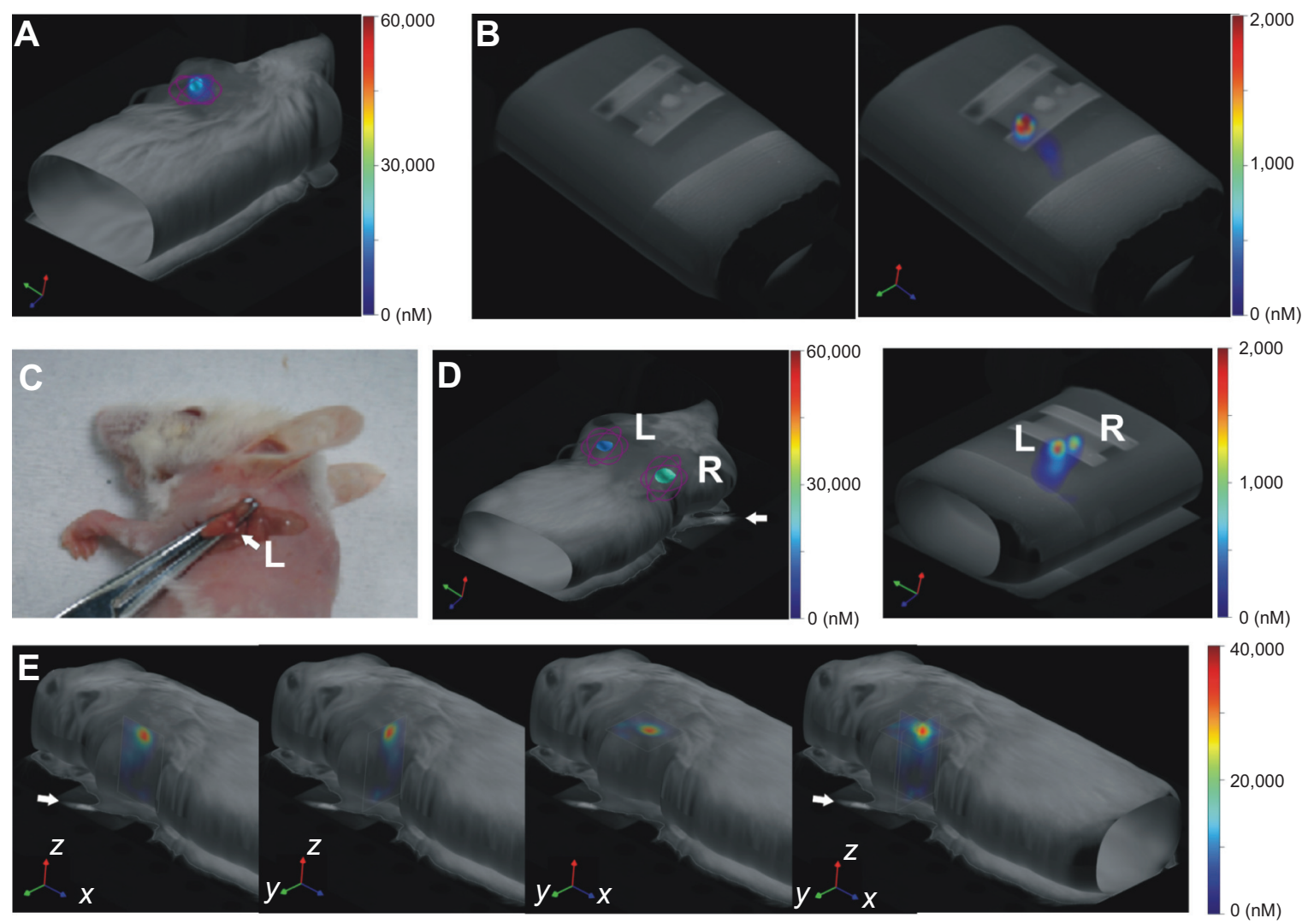

Figure 5 Three-dimensional fluorescence tomographic imaging of lymph node in a mouse using Dex-Cy7 nanogel.

Notes: (A) Typical in vivo fluorescence imaging of lymph node (LN) after intradermal injection of Dex-Cy7 solution into the front left paw of a mouse. (B) Ex vivo imaging of isolated left and right (control) LN. (C) In situ injection of Dex-Cy7 solution in the left LN after removal of mouse skin. (D) In vivo and ex vivo imaging of left and right $\mathrm{LN}$ after intradermal injection of Dex-Cy7 solution into the front right paw of a mouse. (E) Typical tomographic imaging of LN at transverse ( $z$ ) and axial ( $x, y)$ cross-sections and their image combinations 2 hours after intradermal injection.

Abbreviations: L, left; R, right.

and practical LN tracer in a mouse. After injecting Dex-Cy7 nanogel and $\mathrm{MB}$ solution into the left paw of a mouse, the left LN could be detected by the imaging system and, after the removal of mouse skin, the LN was clearly seen in blue (Figure 6A and B). Ex vivo imaging of the left LN again showed that the fluorescence signal was emitted from the LN but not from the surrounding fat tissue (Figure 6C). In addition, in vivo tomographic fluorescence imaging revealed that Dex-Cy7 nanogels were located mainly in the central region of the LN (Figure 5E). These results indicate that Dex-Cy 7 nanogel is effective as a fluorescence nanoprobe for noninvasive mapping of $\mathrm{LN}$ in mouse.

To test whether Dex-Cy7 nanogels have longer retention time in $\mathrm{LN}$ as compared to $\mathrm{Cy} 7$, the accumulation kinetics of the nanogels in LN was studied by in vivo tomographic fluorescence imaging of the left $\mathrm{LN}$ after injection of the nanogel solution into the left paw of a mouse (Figure 7A). On measuring the fluorescence signals in the left LN, the fluorescence intensities first increased from 15 minutes to 110 minutes and then gradually reduced until 3,220 minutes
(Figure 7B). As a control, Cy7 alone offered a shorter retention time (90 minutes) in LN, probably due to its fast diffusion to the adjacent tissue. Besides, Dex-Cy7 nanogels showed stronger fluorescence intensity as compared to $\mathrm{Cy} 7$ alone because they were more photostable than Cy7 (Figure 3F). Furthermore, ex vivo imaging of the harvested organs showed that the nanogels were mainly distributed in the liver and spleen 120 minutes after injection and finally excreted from the organs after 3 days (Figure $7 \mathrm{C}$ ). Besides, $\sim 75 \%$ of the nanogels were cleared from the injection site after 3 days (Figure S5), implying that the nanogels can be eliminated gradually from the mouse body.

\section{Localization and uptake of Dex-Cy7 nanogels in LN}

Further studies were conducted to find the localization of Dex-Cy7 nanogels in LN and their uptake pathway in the cells. To this purpose, immunohistofluorescence (in green) of $\mathrm{LN}$ sections was conducted to stain the macrophages $\left(\mathrm{CD}^{+}\right)$and dendritic cells $\left(\mathrm{CD} 205^{+}\right) 2$ hours after injecting 

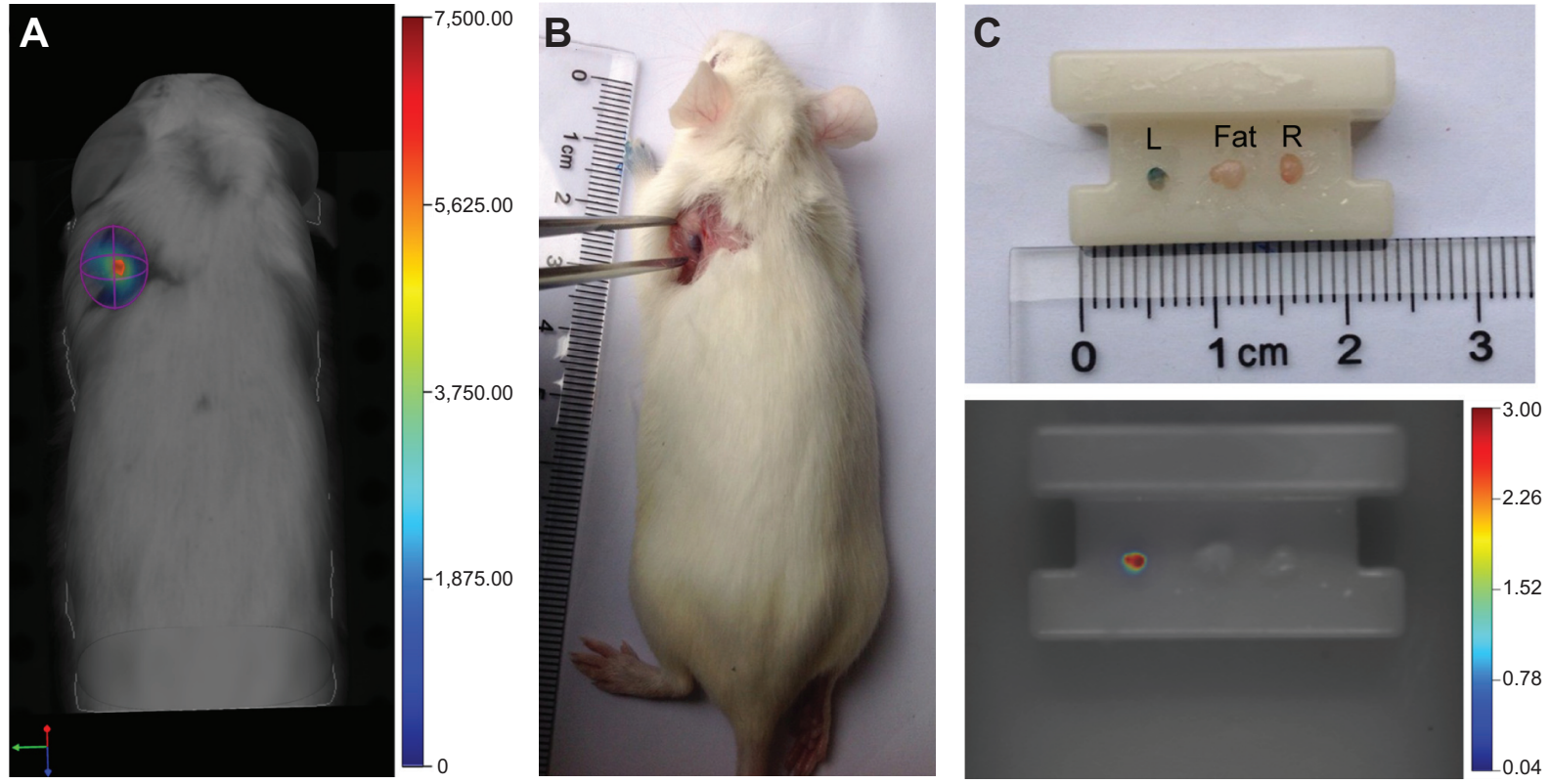

Figure 6 Comparison study between fluorescence imaging and visualization of lymph node in a mouse.

Notes: In vivo imaging (A) and visualization (B) of lymph node (LN) 2 hours after intradermal injection of both Dex-Cy7 and methylene blue solution into the front left paw of a mouse. (C) Ex vivo observation and fluorescence imaging of left $L N(L)$, fat, and right $L N(R)$.

rhodamine B-labeled nanogels (Dex-RB, in red) into the front left paw of a mouse. As shown in Figure 8, the nanogels were found largely in the central area of the LN. This is in good agreement with the result of in vivo tomographic imaging (Figure 5E). Additionally, the merged fluorescence signals (in yellow) confirmed that Dex-RB nanogel uptake occurred predominantly in macrophages rather than in dendritic cells.

Cellular uptake of Dex-RB nanogels was also observed under CLSM after incubating the Dex-RB nanogel with
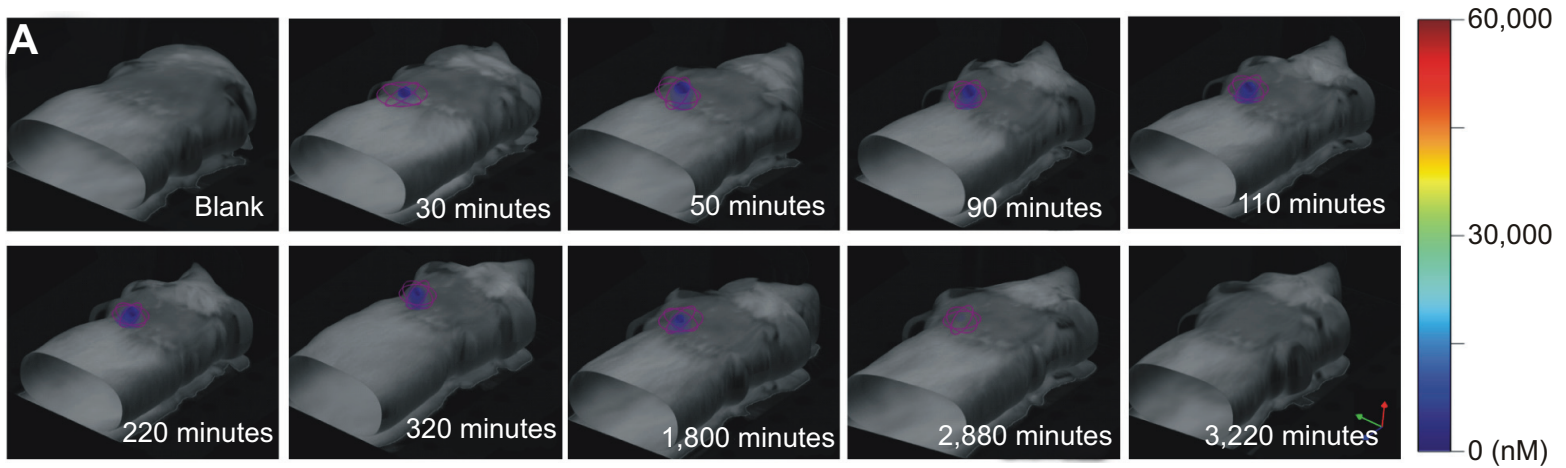

\section{B}

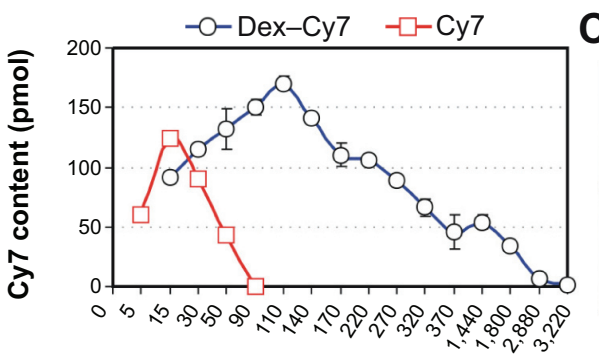

C
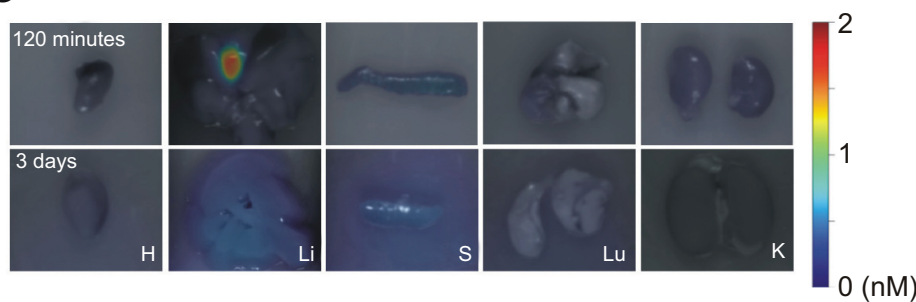

\section{Time (minutes)}

Figure 7 Accumulation kinetics study of Dex-Cy7 nanogel in lymph node in a mouse.

Notes: (A) Accumulation kinetics of Dex-Cy7 in the left lymph node (LN) after intradermal injection and (B) quantified fluorescence signals in the LN for Dex-Cy7 and Cy7 alone (control). (C) Ex vivo imaging of the organs 120 minutes or 3 days after intradermal injection of Dex-Cy7.

Abbreviation: LN, lymph node. 
A
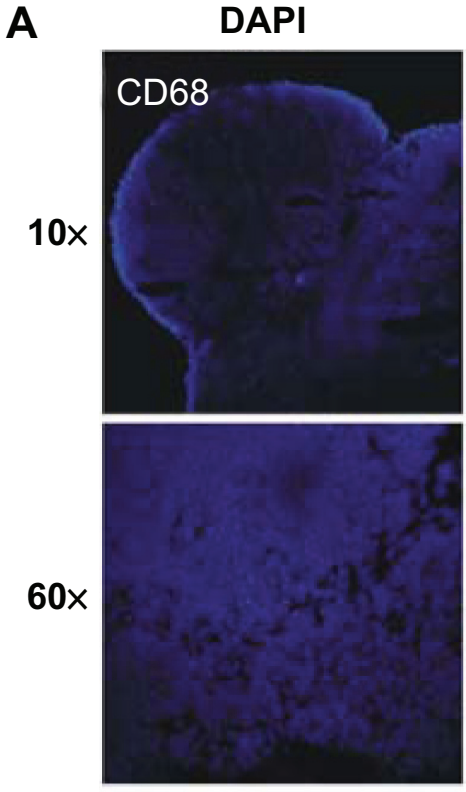

B
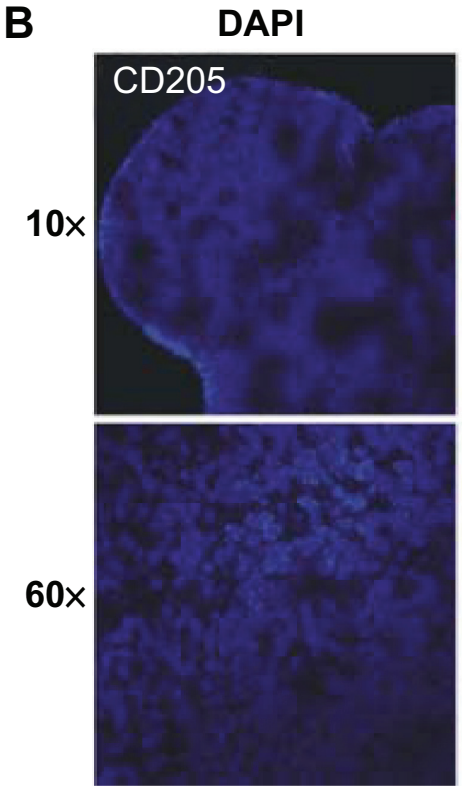
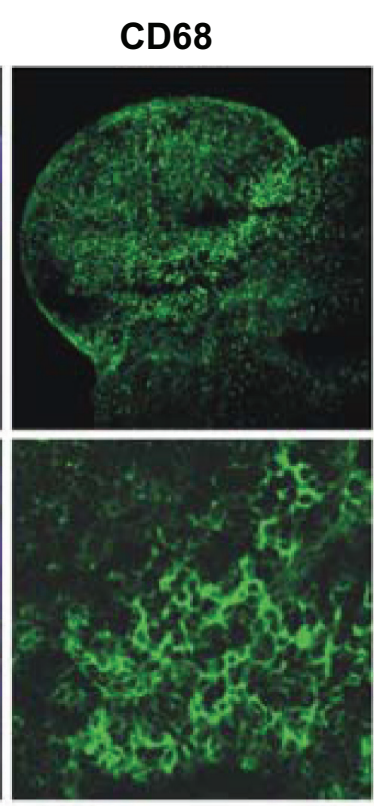

CD205
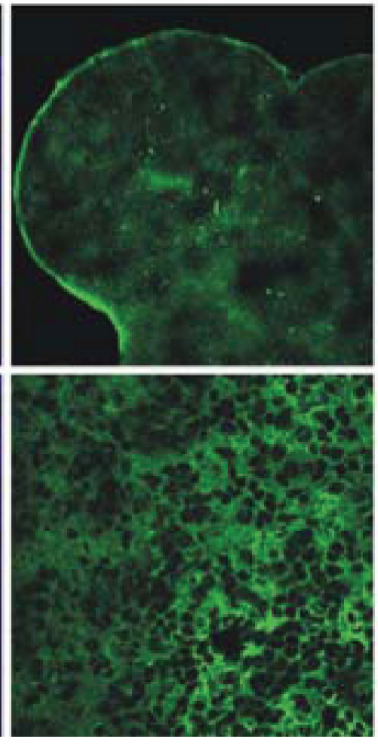

Dex-RB
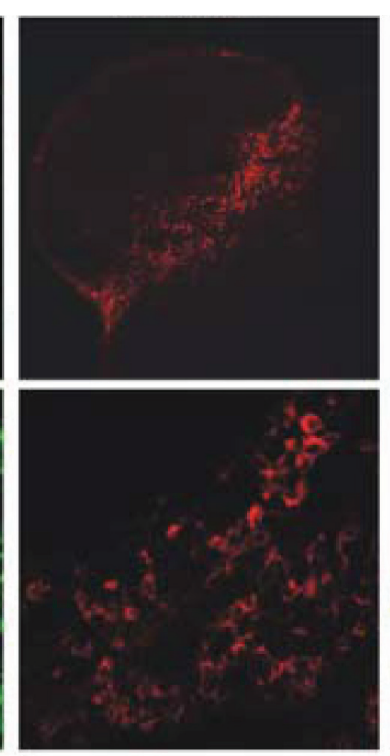

Dex-RB
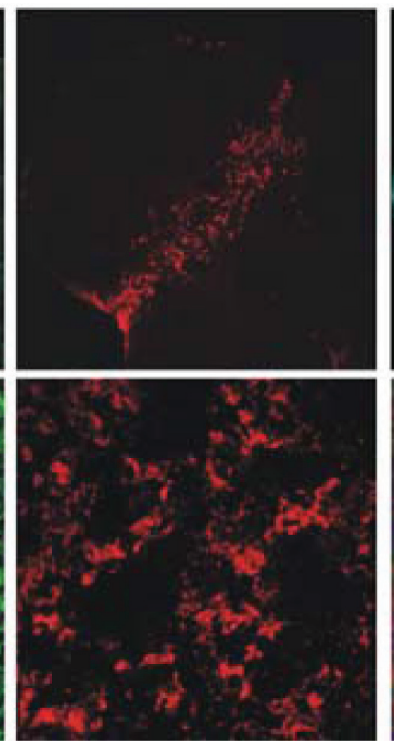

Merge
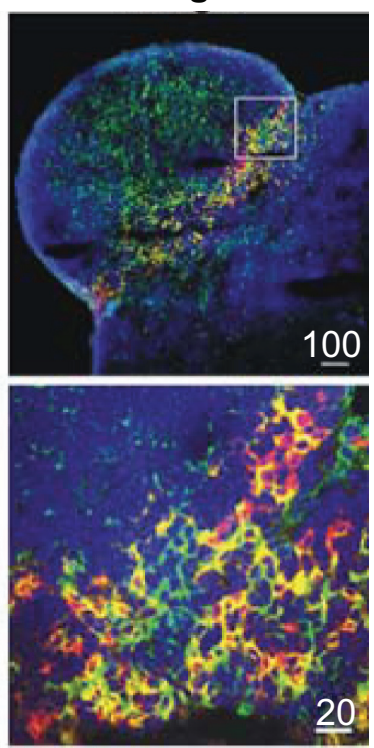

Merge
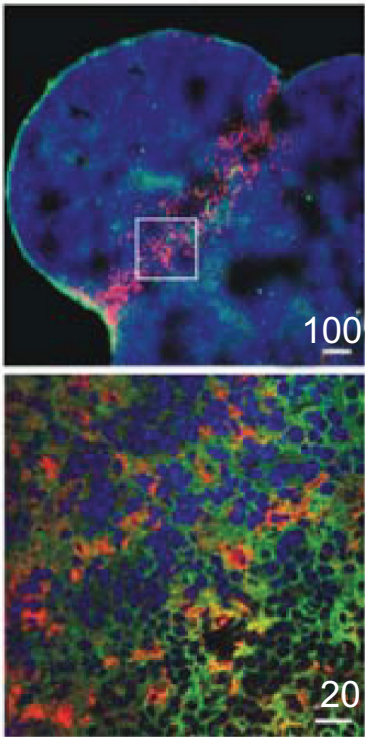

Figure 8 Immunohistofluorescence images of lymph node sections of a mouse 2 hours after intradermal injection of Dex-RB solution. Notes: The sections were stained with anti-CD68 (A) and anti-CD205 (B) and observed at I0× and 60× magnification.

Abbreviation: DAPI, 4',6-diamidino-2-phenylindole dihydrochloride; Dex-RB, rhodamine B-labeled Dex-SS-DCA nanogel.

DC 2.4 cells at different time intervals. As shown in Figure 9, the nanogels were found in lysotracker-staining lysosomes (green) and the cytoplasm 4 hours after uptake. However, the nanogels were mainly localized in the cytoplasm at 6 hours of uptake and finally almost disappeared at 24 hours, suggesting that they undergo biodegradation or excretion by the cells. Inhibitor experiments further showed that chlorpromazin, a clathrin-specific inhibitor, markedly reduced the cellular uptake of Dex-RB nanogels, implying that the nanogels internalize into DC 2.4 cells via a clathrin-mediated uptake (Figure S6).

\section{Discussion and conclusion}

SLN is the first one of a group of LNs for tumor cell drainage from primary tumor. More and more clinical cases have confirmed that SLN biopsy is a critical procedure in the diagnosis of tumor metastasis for the patients of breast cancer. Although clinical SLN tracers are effective for the identification of SLN, they are associated with a few drawbacks including low SLN imaging resolution and invasive modality to human body. In this study, we therefore developed an NIR-emitting nanoprobe based on a dextranbased nanogel as a safe and efficient nanoprobe for SLN 

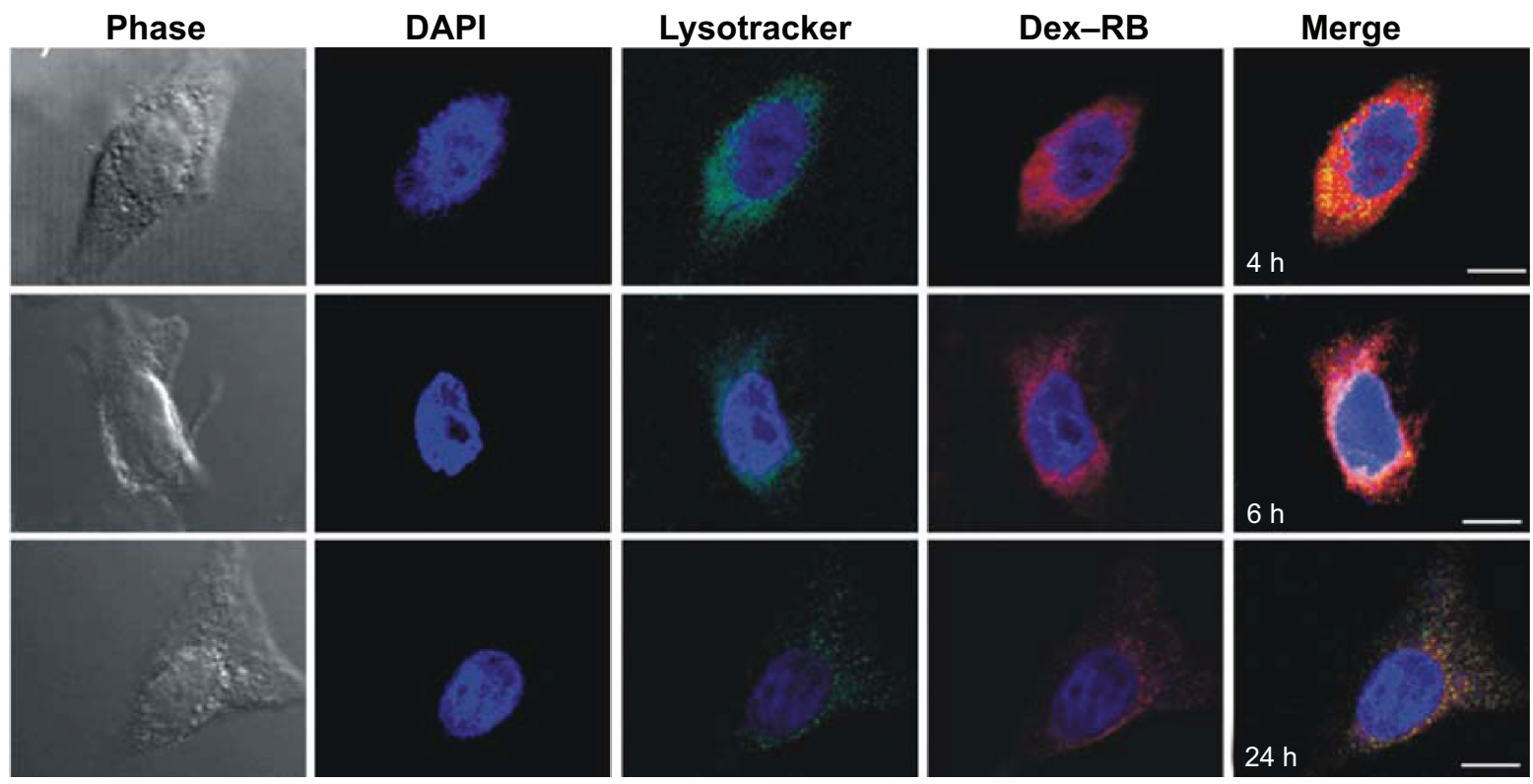

Figure 9 In vitro fluorescence images of Dex-RB accumulated in DC 2.4 cells at different time intervals.

Notes: The lysosomes (in green) were stained with lysotracker.

Abbreviations: DAPI, 4',6-diamidino-2-phenylindole dihydrochloride; h, hours; Dex-RB, rhodamine B-labeled Dex-SS-DCA nanogel.

mapping. NIR fluorescence allows strong light penetration into living tissues that have relatively low auto-fluorescence background. Therefore, NIR fluorescence imaging provides a noninvasive modality for easy and safe SLN mapping. Furthermore, although many inorganic nanoparticles such as QDs and nanogold have been applied for SLN mapping, their controversial biosafety and insufficient data on toxicity in the body remain a big challenge for clinical translation. By contrast, dextran-based nanopartilces have been accepted in the biomedical field, because dextran has few side effects on the organs of human body although it can cause acute renal failure in the patients with chronic renal insufficiency. As a typical example, the use of dextrancoated superparamagnetic iron oxide nanoparticles for magnetic resonance imaging of liver has been approved by the US Food and Drug Administration (FDA). Recently, a new imaging agent based on technetium (Tc) 99-labeled dextran was also approved by the FDA for SLN imaging. However, this agent has the radioactive ${ }^{99} \mathrm{Tc}$ element. Inspired by the research background, we designed the DCA conjugate which can self-assemble to form nanogels. Moreover, in order to achieve biodegradability of the nanogels, the disulfide bond was incorporated as a degradable linker between dextran and DCA. As a result, the nanogels were found to be not only colloidally stable under physiological conditions but also biodegradable inside the cells (Figure S3). In vitro and in vivo toxicity evaluation indicated that the nanogels displayed nontoxicity properties in vitro at nanoparticle concentrations up to $1,000 \mu \mathrm{g} / \mathrm{mL}$, and no adverse effect was observed on liver and kidney functions in vivo at an intravenous injection dose of $1.25 \mathrm{mg} / \mathrm{kg}$ in mice (Figure 4 and S4). Normally, dextran is predominantly excreted in urine within a few days. These results indicate that this biodegradable dextran-based nanogel has good biocompatibility in vivo.

Although a number of polymer-based nanoparticles have been reported for biomedical applications, their usage in SLN mapping is rather limited. A major reason is that most of these nanoparticles have sizes $>50 \mathrm{~nm}$. However, an ideal particle size for the nanoparticles for efficient SLN uptake is in the range $20-50 \mathrm{~nm}^{7}$ To achieve nanoparticles within this size range, we modulated the composition of hydrophilic dextran and hydrophobic DCA. In this way, dextran-based nanogels with the size of $28 \mathrm{~nm}$, which is an optimal size for SLN uptake, could be obtained when the molecular weight of dextran was $10 \mathrm{kDa}$ and the DS of DCA was $\sim 5$ (Figure 1). To our best knowledge, this is the first report on bioreducible nanogels with size below $50 \mathrm{~nm}$. Another property of the dextran-based nanogels for SLN mapping is that they are able to improve the photostability of NIR dyes. Compared to Cy7 alone, Dex-Cy7 displayed superior photostability during 24 hours (Figure 3), implying that the Dex-Cy7 nanogel can emit a stable fluorescence signal for imaging. A rational reason for this improved photostability is that the coupling of $\mathrm{Cy} 7$ to the nanogel minimizes the adverse self-quenching of the $\mathrm{Cy} 7$ molecule. 
The traditional fluorescence imaging system affords a two-dimensional (planar) image. However, for accurate identification of SLNs and their subsequent harvesting, it would be helpful for surgeons to visualize a 3D image of SLN. In our study, we presented fluorescence tomographic images of SLN using the FMT 2500 system. By this system, in vivo 3D fluorescence images of SLN in a mouse was visualized after intradermal injection of the Dex-Cy7 nanoprobe. In addition, the fluorescence tomographic images of the node at the transverse $(z)$ and axial $(x, y)$ cross-sections could be obtained (Figure 5). These additional SLN topographic images would favorably assist surgeons for SLN harvest during operation. Also, ex vivo analysis of isolated LN confirmed that identical LN may be detected by fluorescence tomographic imaging with Dex-Cy7 nanoprobe or visualization with MB, which is a clinical SLN tracer (Figure 6). This suggests the same effectiveness of Dex-Cy7 nanogels and MB.

A unique attribute of this imaging system is real-time quantification of the amount of Dex-Cy7 in SLN, which provides the accumulation kinetics of Dex-Cy7 nanogels in the $\mathrm{LN}$ of a mouse (Figure 7B). The results indicate that Dex-Cy7 nanogels display a longer retention time in the $\mathrm{LN}$ when compared to $\mathrm{Cy} 7$ alone, which is attributed to optimal particle size of the nanogels. Similar to MB, Cy7 has a shorter retention time ( $<90$ minutes) in SLN, which is in part due to its rapid diffusion to the surrounding tissue. Here, fluorescence topographic imaging with the Dex-Cy7 nanoprobe offers useful quantification of data on the imaging of SLN. To our best knowledge, this is the first report on fluorescence topographic imaging of the LN.

Although several nanoparticles such as QDs have been utilized for SLN imaging, it is not well elucidated as to the fate of the nanoparticles in the SLN and their eventual elimination profile in the body. In our study, the Dex-Cy7 nanogel was found to be rapidly eliminated by liver and spleen 3 days after intradermal injection (Figure 7C). In a previous report, Pons et al showed that $\mathrm{CuInS}_{2} / \mathrm{ZnS}$-based QDs were eliminated slowly from mouse 3 months after intradermal injection into the paw of the mouse. ${ }^{19}$ Another work from Erogbogbo et al indicated that silica-based QDs were completely cleaned up by the liver and spleen of the mouse 2 months after intradermal injection. ${ }^{20}$ This slow elimination profile for those inorganic nanoparticles is likely due to their different surface coatings. Obviously, the complicated composition of the inorganic nanoparticles and their slow elimination rate in vivo would give rise to doubts on the biosafety and also be a serious barrier to clinical translation. In fact, Dex-Cy7 nanogels showed much lower in vitro cytotoxicity
$\left(\mathrm{IC}_{50}>100 \mu \mathrm{M}\right)$ as compared to those inorganic nanoprobes such as $\mathrm{CulnS}_{2} / \mathrm{ZnS}$-based QDs $\left(\mathrm{IC}_{50}=100 \mathrm{nM}\right) .{ }^{19}$ Thus, polymeric nanoparticles such as Dex-Cy7 nanogel should be more suitable candidates in the development of an ideal nanoprobe for SLN imaging because of their well-defined structure and composition as well as their good biocompatibility in vivo. However, detailed biosafety of NIR-emitting Dex-Cy7 nanogel must be elucidated in preclinical trials for further clinical translation.

In summary, we have shown that disulfide-linked Dex-SSDCA can be prepared. These conjugates may self-assemble to form nanogels under physiological conditions with nanoscale particle sizes depending on the molecular weight of dextran. The coupling of $\mathrm{Cy} 7$ to the nanogels based on $10 \mathrm{kDa}$ dextran affords NIR-emitting nanogels (Dex-Cy7) with the size of about $28 \mathrm{~nm}$, which is an optimal size for LN uptake. As a result, Dex-Cy7 nanogels display an enhanced photostability and longer retention time in the mouse $\mathrm{LN}$ as compared to $\mathrm{Cy} 7$ itself. The dextran-based nanogels are biodegradable intracellularly, displaying low toxicity and good in vivo biocompatibility. NIR-emitting dextran-based nanogels are promising as safe and effective nanoprobes for the noninvasive mapping of the SLN. In the future work, development of a smart dextranbased nanoprobe will be attempted for the direct diagnosis of SLN status (metastatic or not) instead of the traditional SLN biopsy test. For example, "activatable" molecular imaging probes have been developed for noninvasive diagnosis of tumor tissues. ${ }^{21,22}$ Therefore, a combination of nanoparticles with an activatable molecular imaging probe targeting a lymphangiogenic marker would provide a noninvasive means for both mapping and diagnosis of SLN in the near future.

\section{Acknowledgments}

This work was financially supported by the grants from the Shanghai Science and Technology Committee (124119a4700, ZZ), the Shanghai Health Bureau Scientific Research Subject (20124164, ZZ), the Shanghai Municipal Natural Science Foundation (13ZR1443600, CL) and the National High-Tech R\&D Program of China (2013AA032202).

\section{Author contributions}

Jiejing Li, and Beiqi Jiang worked out all the experimental results. Chao Lin and Zhigang Zhuang contributed the idea of this article. All the authors contributed to writing, data analysis, and discussion.

\section{Disclosure}

The authors report no conflicts of interest in this work. 


\section{References}

1. Karakousis GC, Pandit-Taskar N, Hsu M, et al. Prognostic significance of drainage to pelvic nodes at sentinel lymph node mapping in patients with extremity melanoma. Melanoma Res. 2012;23(1):40-46.

2. Takeuchi H, Kitajima M, Kitagawa Y. Sentinel lymph node as a target of molecular diagnosis of lymphatic micrometastasis and local immunoresponse to malignant cells. Cancer Sci. 2008;99(3):441-450.

3. Lucarelli RT, Ogawa M, Kosaka N, Turkbey B, Kobayashi H, Choyke PL. New approaches to lymphatic imaging. Lymphat Res Biol. 2009;7(4): 205-214.

4. van der Ploeg IM, Russell NS, Nieweg OE, et al. Lymphatic drainage patterns in breast cancer patients who previously underwent mantle field radiation. Ann Surg Oncol. 2009;16(8):2295-2299.

5. Kim TH, Chen Y, Mount CW, Gombotz WR, Li X, Pun SH. Evaluation of temperature-sensitive, indocyanine green-encapsulating micelles for noninvasive near-infrared tumor imaging. Pharm Res. 2010;27(9): 1900-1913.

6. Reddy ST, Rehor A, Schmoekel HG, Hubbell JA, Swartz MA. In vivo targeting of dendritic cells in lymph nodes with poly(propylene sulfide) nanoparticles. J Control Release. 2006;112(1):26-34.

7. Nakajima M, Takeda M, Kobayashi M, Suzuki S, Ohuchi N. Nanosized fluorescent particles as new tracers for sentinel node detection: experimental model for decision of appropriate size and wavelength. Cancer Sci. 2005;96(6):353-356.

8. Frangioni JV. In vivo near-infrared fluorescence imaging. Curr Opin Chem Biol. 2003;7(5):626-634.

9. Ravizzini G, Turkbey B, Barrett T, Kobayashi H, Choyke PL. Nanoparticles in sentinel lymph node mapping. Wiley Interdiscip Rev Nanomed Nanobiotechnol. 2009;1(6):610-623.

10. Kim S, Lim YT, Soltesz EG, et al. Near-infrared fluorescent type II quantum dots for sentinel lymph node mapping. Nat Biotechnol. 2003; 22(1):93-97.

11. Song KH, Kim CH, Cobley CM, Xia YN, Wang LV. Near-infrared gold nanocages as a new class of tracers for photoacoustic sentinel lymph node mapping on a rat model. Nano Lett. 2009;9(1):183-188.
12. Noh YW, Kong SH, Choi DY, et al. Near-infrared emitting polymer nanogels for efficient sentinel lymph node mapping. ACS Nano. 2012; 6(9):7820-7831.

13. Melancon MP, Wang Y, Wen X, et al. Development of a macromolecular dual-modality MR-optical imaging for sentinel lymph node mapping. Invest Radiol. 2007;42(8):569-578.

14. Meng F, Hennink WE, Zhong Z. Reduction-sensitive polymers and bioconjugates for biomedical applications. Biomaterials. 2009;30(12): 2180-2198.

15. Zugates GT, Anderson DG, Little SR, Lawhorn IEB, Langer R. Synthesis of poly(beta-amino ester)s with thiol-reactive side chains for DNA delivery. J Am Chem Soc. 2006;128(39):12726-12734.

16. Lin C, Zhao P, Li F, Guo F, Li Z, Wen X. Thermosensitive in situforming dextran-pluronic hydrogels through Michael addition. Mater Sci Eng C. 2010;30(8):1236-1244.

17. LiD, Tang X, Pulli B, et al. Theranostic nanoparticles based on bioreducible polyethylenimine-coated iron oxide for reduction-responsive gene delivery and magnetic resonance imaging. Int J Nanomedicine. 2014;9: 3347-3361.

18. Song Y, Lou B, Zhao P, Lin C. Multifunctional disulfide-based cationic dextran conjugates for intravenous gene delivery targeting ovarian cancer cells. Mol Pharm. 2014;11(7):2250-2261.

19. Pons T, Pic E, Lequeux N, et al. Cadmium-free $\mathrm{cuInS}_{2} / \mathrm{ZnS}$ quantum dots for sentinel lymph node imaging with reduced toxicity. ACS Nano. 2010;4(5):2531-2538.

20. Erogbogbo F, Yong KT, Roy I, et al. In vivo targeted cancer imaging, sentinel lymph node mapping and multi-channel imaging with biocompatible silicon nanocrystals. ACS Nano. 2011;5(1):413-423.

21. Chen K, Chen XY. Design and development of molecular imaging probes. Curr Top Med Chem. 2010;10(12):1227-1236.

22. Weissleder $\mathrm{R}$, Tung $\mathrm{CH}$, Mahmood $\mathrm{U}$, Bogdanov A. In vivo imaging of tumors with protease-activated near-infrared fluorescent probes. Nat Biotechnol. 1999;17(4):375-378. 


\section{Supplementary materials}

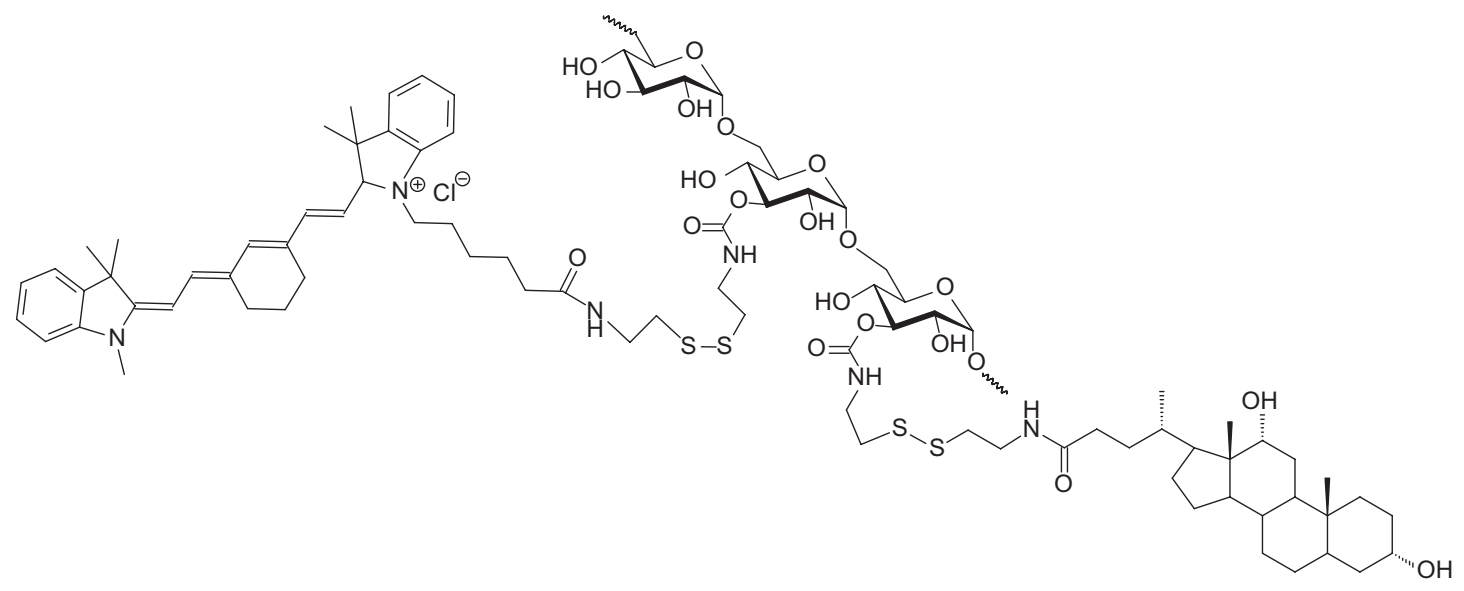

Figure SI Chemcial structure of Cy7-labeled Dex-SS-DCA conjugate (Dex-Cy7).

Abbreviation: Dex-SS-DCA, dextran-deoxycholic acid conjugate.

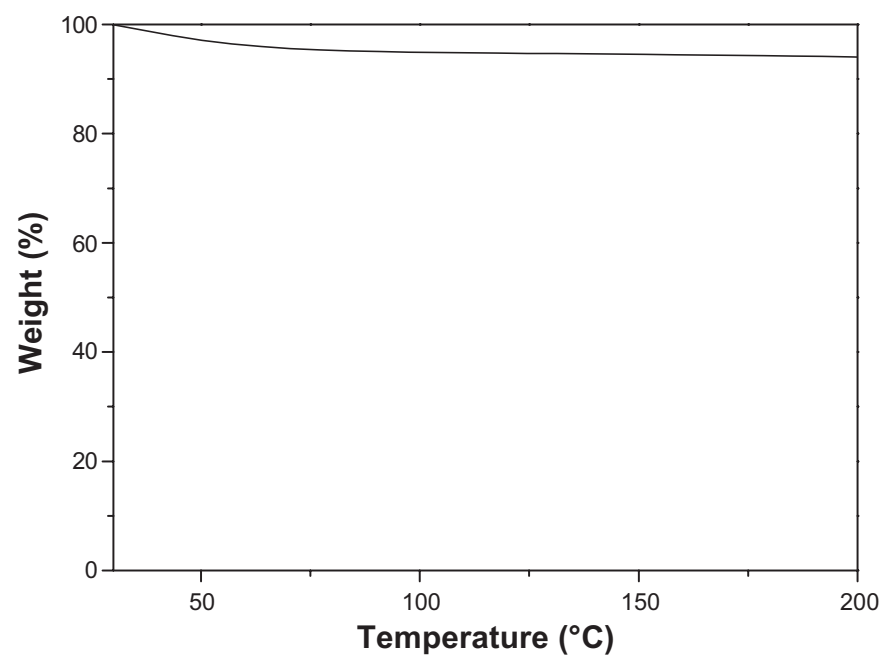

Figure S2 Thermogravimetric analysis curve of Dex 10k-SS-DCA nanogels.

Abbreviation: Dex-SS-DCA, dextran-deoxycholic acid conjugate.

A

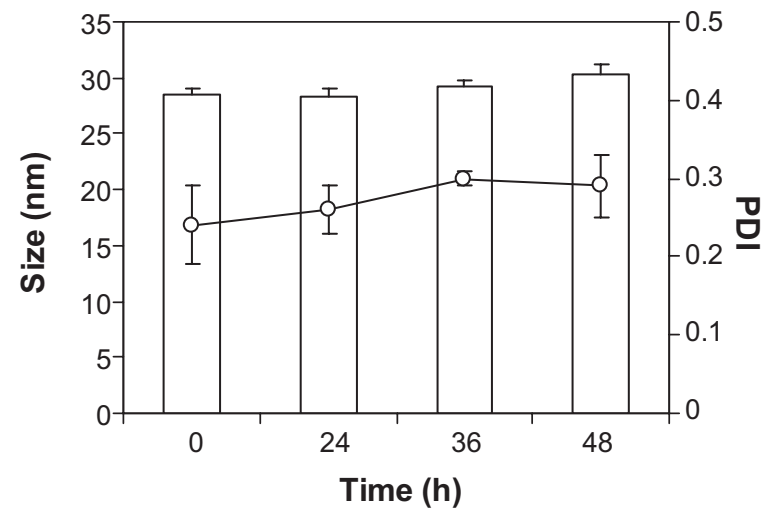

B $\quad-\infty$ w/o DTT $\quad-$ w/DTT

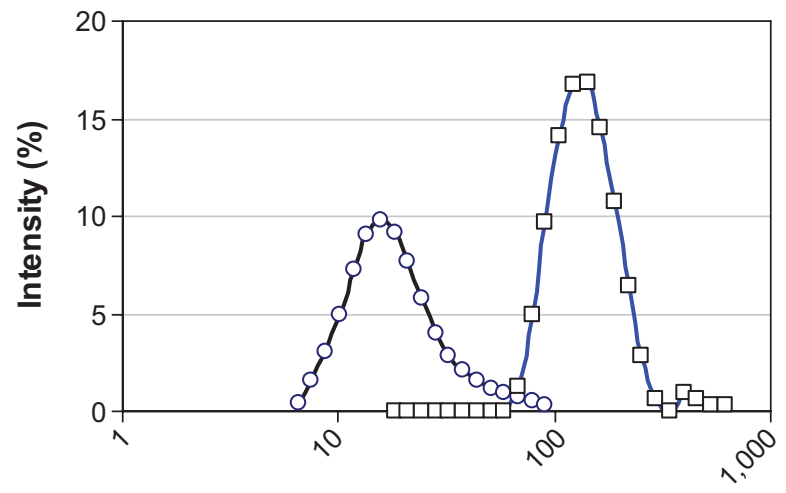

Figure S3 Dynamic light scattering analysis of the average particle size of Dexl0k-SS-DCA nanogels as a function of time (A) and size distribution (B) of the nanogels in the absence (w/o) or presence ( $w /)$ of $2.5 \mathrm{mM}$ dithiothreitol.

Abbreviations: Dex-SS-DCA, dextran-deoxycholic acid conjugate; PDI, polydispersity index; DTT, dithiothreitol; h, hours. 


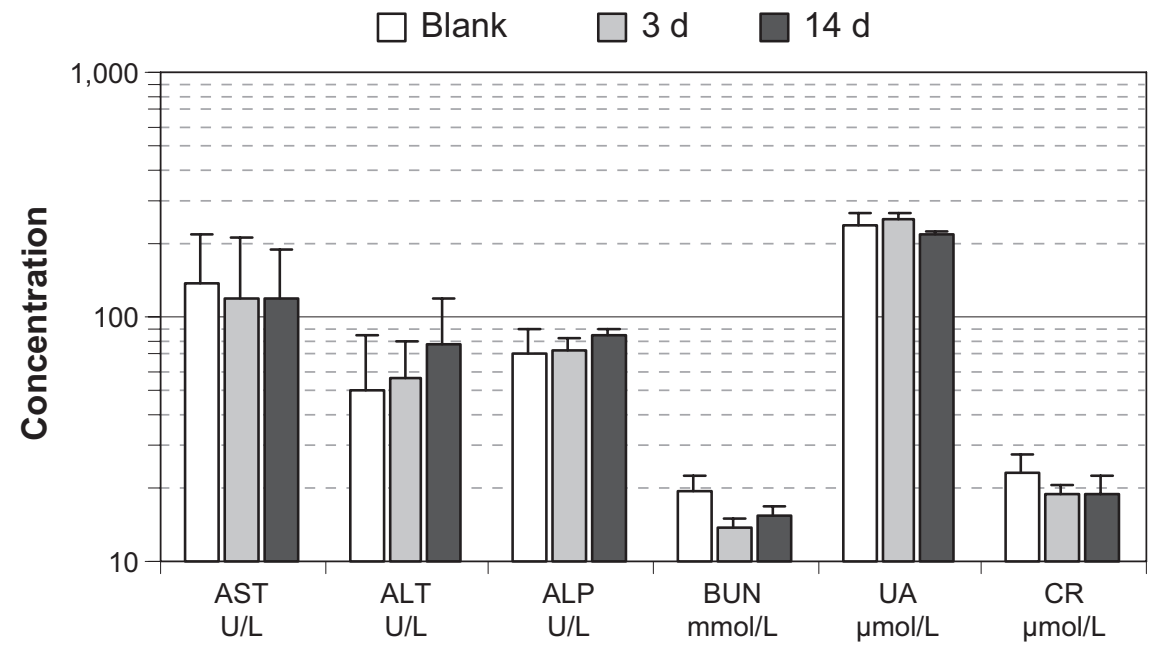

Figure S4 Liver and kidney function assay 3 days or 14 days after intravenous injection of Dex-Cy7 nanogels at a dose of I.25 mg/kg in mice.

Abbreviations: AST, aspartate transaminase; ALT, alanine transaminase; ALP, alkaline phosphatase; BUN, blood urea nitrogen; UA, uric acid; d, days; CR, creatinine clearance rate; $\bigcup$, unit.

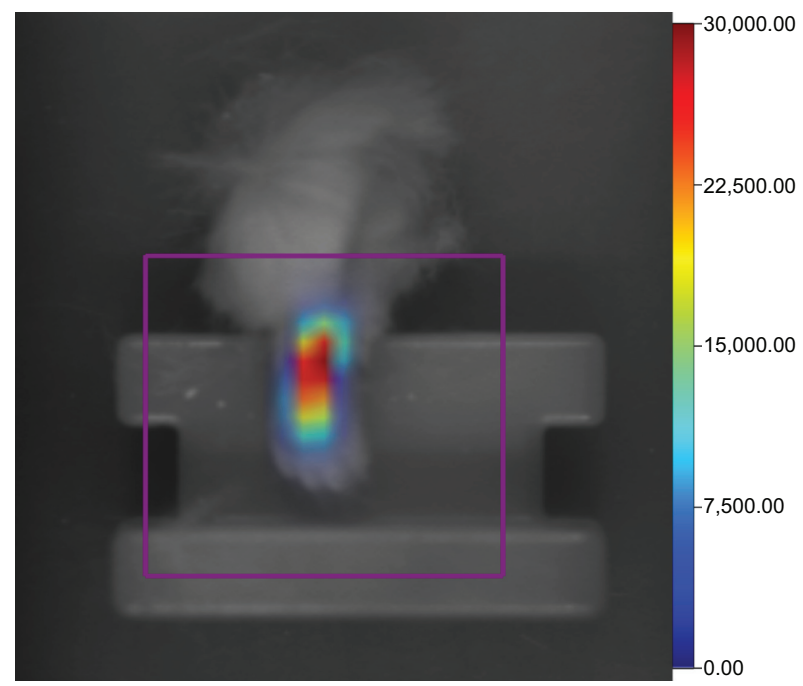

Figure S5 In vivo fluorescence imaging of Dex-Cy7 at the injection site 3 days after intradermal injection.

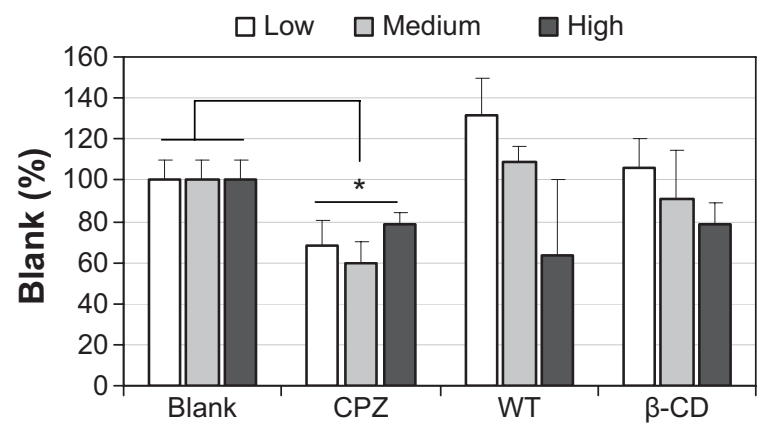

Figure S6 Inhibiting study on cellular uptake of Dex-RB nanogel in DC 2.4 cells at different (low, medium, high) inhibitor concentrations, ie, $14 \mu \mathrm{M}, 28 \mu \mathrm{M}$, and $56 \mu \mathrm{M}$ of CPZ, $12.5 \mu \mathrm{M}, 25 \mu \mathrm{M}$, and $50 \mu \mathrm{M}$ of WT and $40 \mu \mathrm{M}, 80 \mu \mathrm{M}$, and $\mathrm{I} 60 \mu \mathrm{M}$ of $\beta-C D$.

Note: It was verified that at these concentrations the inhibitors had no cytotoxicity against DC 2.4 cells. $* P<0.05$

Abbreviations: $C P Z$, chlorpromazine; $W T$, wortamannin; $\beta-C D, \beta$-cyclodextrin.
International Journal of Nanomedicine

\section{Publish your work in this journal}

The International Journal of Nanomedicine is an international, peerreviewed journal focusing on the application of nanotechnology in diagnostics, therapeutics, and drug delivery systems throughout the biomedical field. This journal is indexed on PubMed Central, MedLine, CAS, SciSearch ${ }^{\circledR}$, Current Contents ${ }^{\circledR} /$ Clinical Medicine,

\section{Dovepress}

Journal Citation Reports/Science Edition, EMBase, Scopus and the Elsevier Bibliographic databases. The manuscript management system is completely online and includes a very quick and fair peer-review system, which is all easy to use. Visit http://www.dovepress.com/ testimonials.php to read real quotes from published authors. 